\title{
Synbiotic (Lactobacillus Pentosus GSSK2 And Isomalto-Oligosaccharides) Supplementation Modulates Pathophysiology And Gut Dysbiosis In Experimental Metabolic Syndrome
}

\author{
Sakshi Khanna \\ Panjab University \\ Kanthi Kiran Kondepudi \\ National Agri-food Biotechnology Institute (NABI), S.A.S. Nagar \\ Mahendra Bishnoi \\ National Agri-food Biotechnology Institute (NABI), S.A.S. Nagar \\ Geeta Shukla ( $\sim$ geeta_shukla@pu.ac.in ) \\ Panjab University
}

\section{Research Article}

Keywords: disease, microbiota, syndrome, histomorphology, prodigious

Posted Date: May 19th, 2021

DOl: https://doi.org/10.21203/rs.3.rs-529631/v1

License: (c) (i) This work is licensed under a Creative Commons Attribution 4.0 International License.

Read Full License 
$5 \quad{ }^{1}$ Department of Microbiology, Panjab University, Chandigarh, India

$6 \quad{ }^{2}$ National Agri-food Biotechnology Institute (NABI), S.A.S. Nagar, Punjab, India

7

8 *Corresponding author

9 Professor Geeta Shukla

10

11 modulates pathophysiology and gut dysbiosis in experimental metabolic syndrome

Sakshi Khanna ${ }^{1}$, Kanthi Kiran Kondepudi ${ }^{2 *}$, Mahendra Bishnoi ${ }^{2}$, Geeta Shukla ${ }^{{ }^{*}}$

Department of Microbiology, Basic Medical Sciences Block A, South Campus, Panjab

University, Chandigarh, 160014, India. Email:geeta_shukla@pu.ac.in

ORCID: 0000000212122211

\section{Dr. Kanthi Kiran Kondepudi}

Healthy Gut Research Group, Food \& Nutrition Biotechnology Division, National Agri-Food Biotechnology Institute (NABI), S.A.S. Nagar, Punjab 140306, India. Email: kiran@ nabi.res.in

政

7

8

9

0

1

5

Synbiotic (Lactobacillus pentosus GSSK2 and isomalto-oligosaccharides) supplementation 


\section{Abstract}

Metabolic syndrome a lifestyle disease, where diet and gut microbiota play a prodigious role in its initiation and progression. Prophylactic bio-interventions employing probiotics and prebiotics offer an alternate nutritional approach towards attenuating its progression. The present study aimed to evaluate the protective efficacy of a novel synbiotic (L. pentosus GSSK2 + isomaltooligosaccharides) in comparison to orlistat in an experimental model of metabolic syndrome. It was observed that supplementation of synbiotic for 12 weeks to Sprague Dawley rats fed with high fat diet, ameliorated the anthropometric parameters i.e. weight gain, abdominal circumference, Lee's index, BMI and visceral fat deposition along with significantly increased fecal Bacteroidetes to Firmicutes ratio, elevated population of Lactobacillus spp., Akkermansia spp., Faecalibacterium spp., Roseburia spp. and decreased Enterobacteriaceae compared with HFD animals. Additionally, synbiotic administration to HFD animals exhibited improved glucose clearance, lipid biomarkers, alleviated oxidative stress, prevented leaky gut phenotype, reduced serum lipopolysaccharides and modulated the inflammatory, lipid and glucose metabolism genes along with restored histomorphology of adipose tissue, colon and liver compared with HFD animals. Taken together, the study highlights the protective potential of synbiotic in comparison with its individual components in ameliorating HFD-induced metabolic complications.

\section{Introduction}

Metabolic syndrome, which encompasses obesity, dyslipidemia, hyperglycemia and chronic inflammation in metabolic tissues has emerged as a public health challenge affecting about one quarter of the world's population and the prevalence is predicted to escalate in developed, developing and under developed countries ${ }^{1}$. Inclination towards high-energy diet and decreased physical activity have led to the increased prevalence of metabolic syndrome globally, and is associated with several pathophysiological alterations such as weight gain, ectopic fat deposition, hyperlipidemia, insulin resistance and alterations in gut microbiota, referred as the "second genome" $"$.3. Moreover, gut dysbiosis associated with high calorie intake, leads to significant loss of microbial diversity, increased energy harvest, disruption of gut barrier integrity, low grade inflammation resulting into metabolic endotoxemia, production of reactive oxygen species and deregulation of genes involved in lipid, glucose metabolism and inflammation which play a 
prodigious role in the advancement of metabolic complications ${ }^{4}$. Therefore, development of gut microbiota targeted strategy employing probiotics, prebiotics and synbiotics, that could potentially re-establish the gut homeostasis is budding as the novel prophylactic biointervention for alleviating metabolic disorders.

Probiotics are 'live microorganisms that when administered in adequate amounts, confer a health benefit on the host' and the health promoting potentials include maintenance of gut homeostasis, alienating pathogens, enhancing the bioavailability of nutrients, stimulation and modulation of host immune system ${ }^{5,6}$. Due to their multifarious benefits, probiotics are currently the major focus of attention to be explored as potential biotherapeutics for the management of various gastrointestinal ailments, liver damage, cancers, inflammatory and metabolic disorders ${ }^{7,8}$. Prebiotics, are non-digestible food ingredients that selectively stimulate the growth or activity of beneficial microorganisms in the host ${ }^{9}$. Biofermentation of prebiotic fibres releases short chain fatty acids, which are the messengers of cross talk between gut microbiota and host thereby regulating intestinal inflammatory response and colon health ${ }^{10}$.

Synbiotic, combination of probiotic with prebiotic, has been found to have a synergistic effect on host health and various experimental studies have indicated the protective and stimulatory effect of synbiotics ${ }^{11,12}$. Recently, we have observed that oral supplementation of probiotic isolate L. pentosus GSSK2 ameliorated the adiposity parameters and various biochemical components of metabolic syndrome and the probiotic isolate was found to metabolize isomaltooligosaccharides (IMOs), a prebiotic reported to possess protective effects in an array of ailments by modulating immune response, improving gut flora, regulating carbohydrate and lipid metabolism 13-15. Though some studies have reported that synbiotics can help in alleviating obesity and related complications, but no information is available with reference to the combination of probiotic with IMOs as synbiotic in ameliorating diet induced metabolic syndrome. Therefore, the need of hour is to explore novel biointerventions that are safer yet effective for the management of metabolic syndrome to overcome the adverse effects of commonly prescribed weight loss drugs like orlistat ${ }^{16}$. Thus, the present study aimed to evaluate the protective efficacy of a novel synbiotic (L. pentosus GSSK2 + IMOs) in comparison to orlistat in experimental model of metabolic syndrome.

\section{Results}




\section{Improved anthropometric parameters and reduced adiposity}

87 It was interesting to observe that animals fed either with synbiotic+HFD (Group VIII) or orlistat+HFD (Group IX) had significantly $(\mathrm{p}<0.05)$ reduced body and liver weight, adipose tissue weight, abdominal circumference, BMI and Lee's index followed by L. pentosus GSSK2+ HFD (Group IV) and IMOs+HFD (Group VI) compared with HFD (Group II) animals (Fig. 1a, b, c, d \& e). However, animals belonging to either probiotic (Group III), prebiotic (Group V) or synbiotic (Group VII) had adiposity parameters comparable to control (Group I) and average feed intake was almost similar in animals belonging to all the groups (Group I-IX, Fig. 1f). Interestingly, gross macroscopic examination of animals belonging to synbiotic+HFD (Group VIII) showed minimum fat deposits in adipose tissue, followed by orlistat+HFD (Group IX), $L$. pentosus GSSK2+HFD (Group IV) and IMOs+HFD (Group VI) compared with HFD animals (Group II, Fig. S1 a to i).

Improved glucose tolerance

It was observed that administration of either probiotic L. pentosus GSSK2 (Group IV), synbiotic (Group VIII) or orlistat (Group IX) to HFD animals significantly ( $<<0.05)$ lowered the fasting blood glucose level and enhanced glucose tolerance compared with HFD (Group II) animals that had increased fasting blood glucose level and impaired glucose clearance from circulation as depicted by an increase in the AUC during OGTT (Fig. 2a, b \& c). However, animals belonging to IMOs+HFD (Group VI) did not show any significant improvement in glucose tolerance while animals supplemented either with probiotic (Group III), prebiotic (Group V) or synbiotic (Group VII) had blood glucose parameters comparable to control (Group I, Fig. 2a, b \& c). Increased LAB count and lipids in feces

The LAB count in feces, an indicator of healthy gut, increased significantly $(\mathrm{p}<0.05)$ in animals belonging to synbiotic, probiotic and prebiotic inspite of HFD feeding compared with control (Group I) and HFD (Group II) animals but maximum increase in LAB was in synbiotic (Group VII) animals (Figure 2d). Further, it was observed that supplementation of synbiotic led to increased fecal lipid excretion inspite of HFD, compared with counter controls (Group II, IV, VI,

\section{Improved gut bacteria composition}

115 Supplementation of synbiotic to HFD animals (Group VIII) led to maximum increase in the 
HFD (Group VI) and orlistat+HFD (Group IX) respectively, compared with HFD (Group II) animals (Fig. 3a, b \& c). Further, animals belonging to synbiotic+HFD (Group VIII) had significantly $(\mathrm{p}<0.05)$ elevated population of Lactobacillus spp., Akkermansia spp., Faecalibacterium spp., and Roseburia spp. while L. pentosus GSSK2+HFD (Group IV) had significantly $(\mathrm{p}<0.05)$ high number of Lactobacillus spp. and Roseburia spp. while IMOs+HFD animals (Group VI) had increased population of Lactobacillus spp. and Faecalibacterium spp whereas, orlistat+ HFD (Group IX) animals did not show any significant change in any of the bacterial genera compared with HFD animals (Figure 3d). Moreover, Enterobacteriaceae population decreased significantly $(\mathrm{p}<0.05)$ in synbiotic + HFD (Group VIII), L. pentosus GSSK2+HFD (Group IV), IMOs+ HFD (Group VI) and orlistat+HFD (Group IX) animals compared with HFD animals (Figure 3d). Further, it was found that animals administered with probiotic (Group III) had increased abundance of Lactobacillus spp. and Roseburia spp., prebiotic (Group V) animals had increased Lactobacillus spp., Akkermansia spp, Faecalibacterium spp and Ruminococcus spp. while synbiotic (Group VII) supplemented animals had increased population of Lactobacillus spp., Akkermansia spp, Faecalibacterium spp, Roseburia spp., and Ruminococcus spp. compared with control (Group I) animals (Figure 3d).

\section{Improved serum biomarkers}

It was found that though animals belonging to synbiotic+ HFD (Group VIII) and orlistat+HFD (Group IX) had significantly ( $\mathrm{p}<0.05$ ) reduced obesity associated serum biomarkers i.e. total cholesterol, triglycerides and LDL cholesterol with increased HDL cholesterol while L. pentosus GSSK2+HFD (Group IV) animals had significantly ( $p<0.05$ ) reduced levels of triglycerides and LDL-cholesterol and IMOs+HFD (Group VI) had reduced levels of triglycerides compared with HFD animals (Group II, Table 1).

Further, L. pentosus GSSK2+HFD (Group IV), synbiotic (Group VIII) and orlistat (Group IX) supplementation to HFD animals significantly $(\mathrm{p}<0.05)$ reduced bilirubin, AST and ALT levels while IMOs+HFD (Group VI) animals showed significant reduction in bilirubin compared with HFD (Group II) animals (Table 1).

It was also observed that synbiotic+ HFD (Group VIII) and orlistat (Group IX) animals had significantly $(\mathrm{p}<0.05)$ reduced levels of serum LPS, TNF- $\alpha$ and IL-6 followed by L. pentosus GSSK2+ HFD (Group IV) and IMOs+ HFD (Group VI) animals compared with their elevated levels in serum of HFD (Group II) animals (Table 1). 


\section{Enhanced antioxidant and suppressed oxidant level}

It was interesting to observe that supplementation of synbiotic to HFD animals (Group VIII) led to significant $(\mathrm{p}<0.05)$ reduction in oxidant MDA level both in adipose tissue and colon whereas orlistat+HFD (Group IX) animals had maximum reduction of MDA in colon followed by $L$. pentosus GSSK2+HFD (Group IV) and IMOs+HFD (Group VI) respectively compared with HFD animals (Table 2). Further, antioxidant GSH and SOD level increased in adipose tissue, colon as well as liver of synbiotic+HFD (Group VIII), followed by L. pentosus GSSK2+HFD (Group IV) and orlistat+HFD (Group IX) while IMOs+HFD (Group VI) animals did not show significant $(\mathrm{p}<0.05)$ change in GSH and SOD level compared with HFD animals (Table 2).

\section{Modulation of gene expression}

It was found that daily administration of synbiotic (Group VIII) and orlistat (Group IX) to HFD animals significantly $(\mathrm{p}<0.05)$ downregulated the expression of genes involved in lipid and glucose metabolism (FASN, HSL, GLUT-4 and glucokinase) and inflammatory markers (TNF- $\alpha$ and IL-6) in liver compared with HFD animals (Group II, Figure 4a). However, animals belonging to L. pentosus GSSK2+HFD (Group IV) had significantly $(\mathrm{p}<0.05)$ decreased expression of FASN, TNF- $\alpha$ and IL-6 while FASN, TNF- $\alpha$ and GLUT-4 were downregulated in IMOs+ HFD (Group VI) animals (Figure 4a).

The expression of adiposity genes, $\mathrm{C} / \mathrm{EBP} \alpha$ and $\operatorname{PPAR} \gamma$ in adipose tissue was significantly $(\mathrm{p}<0.05)$ downregulated in orlistat+HFD (Group IX) followed by synbiotic+HFD (Group VIII), IMOs+ HFD (Group VI) and L. pentosus GSSK2+ HFD (Group IV) respectively compared with HFD (Group II) animals while adiponectin expression was significantly $(\mathrm{p}<0.05)$ upregulated in synbiotic+HFD (Group VIII) and L. pentosus GSSK2+HFD (Group IV) compared with HFD (Group II) animals (Figure 4b). Moreover, the expression of adipokine gene i.e. leptin and inflammatory genes i.e. TNF- $\alpha$ and IL-6 was significantly $(\mathrm{p}<0.05)$ downregulated in synbiotic+HFD (Group VIII) and orlistat+HFD (Group IX) followed by L. pentosus GSSK2+HFD (Group IV) and IMOs+HFD animals (Group VI) compared with HFD animals (Group II, Figure 4b).

The expression of gut integrity gene, claudin in colon was significantly $(\mathrm{p}<0.05)$ upregulated in synbiotic+HFD (Group VIII) and orlistat+HFD (Group IX) while expression of Muc-2 was upregulated in L. pentosus GSSK2+HFD (Group IV) and synbiotic+HFD (Group VIII) 
compared with HFD animals (Group II). Further, synbiotic+HFD animals (Group VIII) had maximum downregulation of TLR-4 expression followed by L. pentosus GSSK2+HFD (Group IV), orlistat+HFD (Group IX) and IMOs+HFD (Group VI) animals respectively while there was no significant change in $\mathrm{CDX}-2$ expression in animals belonging to various groups compared with HFD (Group II) animals (Figure 4c).

\section{Histological modulation}

Histological analysis of adipose tissue of HFD (Group II) animals showed hypertrophied adipocytes indicated by increased mean adipocyte size compared with normal histoarchitecture of adipocytes of control (Group I) animals (Fig. 5a, b \& j). Interestingly, supplementation of probiotic, prebiotic, synbiotic and orlistat to HFD animals for 12 weeks led to reduced adipocyte hypertrophy. Maximum reduction in mean adipocyte size was observed in synbiotic+HFD (Group VIII) followed by orlistat+HFD (Group IX), L. pentosus GSSK2+HFD (Group VI) and IMOs+HFD (Group VI) animals respectively (Fig. 5d, f, h, i \& j). However, adipose tissue of animals belonging to either probiotic (Group III), prebiotic (Group V), or synbiotic (Group VII) had normal histoarchitecture of adipocytes (Fig. 5c, e, g).

The morphological examination of liver of animals belonging to HFD (Group II) showed ballooning degeneration of hepatocytes and hypertrophy compared with normal hepatocyte of probiotic (Group III), prebiotic (Group V), synbiotic (Group VII) and control (Group I) animals (Fig. 6a \& b). However, supplementation of either probiotic (Group IV), synbiotic (Group VIII) or orlistat (Group IX) to HFD animals led to reduced hepatic steatosis along with minimal fat deposition in hepatocytes compared with HFD (Group II) animals whereas, IMOs+HFD (Group VI) animals had increased hepatic steatosis with ballooned hepatocytes (Fig. 6c, d, e, f, g, h \& i).

The colon segments of HFD (Group II) animals showed disrupted crypts, focal colitis in the form of excess lymphocytes between glands and hyperplasia compared with normal and intact mucosal epithelium of probiotic (Group III), prebiotic (Group V), synbiotic (Group VII) and control (Group I) animals (Fig. 7 a, b, c, e \& g). Interestingly, the colon of L. pentosus GSSK2+ HFD (Group IV), synbiotic+HFD (Group VIII) and orlistat+HFD (Group IX) too had intact epithelium lining and closely packed mucus glands with minimal infiltration of inflammatory cells compared with focal accumulation of lymphocytes in the colonic mucosa of IMOs+HFD (Group VI) animals (Fig. 7 d, f, h \& i). 


\section{Discussion}

209 Trialogue between diet, gut microbiota and host immune response has revealed novel prophylactic interventions employing probiotics and prebiotics for metabolic diseases. In our earlier studies, we have observed that indigenous potential probiotic L. pentosus GSSK2 showed potent anti-inflammatory activity in LPS induced RAW 264.7 cells, metabolized prebiotic IMOs and ameliorated adiposity parameters and overall body physiology in HFD fed SD rats ${ }^{14,15}$. In

214 present study, an attempt was made to investigate the prophylactic potential of a novel synbiotic 215 biointervention (Lactobacillus pentosus GSSK2 + IMOs) in experimental metabolic syndrome compared with orlistat, a commonly used weight loss drug.

217 Increased body weight is one of the important hallmark of metabolic syndrome and is 218 accompanied by fat mass deposition. Interestingly, synbiotic+HFD animals had improved adiposity parameters with no change in average feed intake which might be attributed to lipoprotein lipase inhibition and release of appetite-reducing hormones glucagon-like peptide-1 and peptide YY either by probiotic or its metabolites, increased abundance of gut bacteria inhibiting fatty acid uptake in adipose tissue resulting into increased energy expenditure, reduced visceral adipose tissue deposits and decreased mean adipocyte size ${ }^{17,18}$. Esposito et al. ${ }^{19}$ have also observed that supplementation of VSL\#3 to HFD fed rats reduced fat mass with equal food intake, while Liu et al. ${ }^{20}$ documented that administration of probiotic L. plantarum ZJUFT17 to HFD mice had reduced weight gain due to decreased feed intake.

227 Hyperglycemia, a key outcome of metabolic syndrome, was ameliorated in synbiotic+HFD 228 animals, suggesting improved glucose metabolism and is in concordance with earlier study ${ }^{21}$. 229 These scientists have demonstrated that probiotic L. sakei OK67 reduced the blood glucose levels in HFD-fed mice due to decrease in LPS producing Gram negative gut bacteria and preventing LPS induced inflammation resulting into alleviated $\beta$ cell dysfunction ${ }^{22}$. The 232 antidiabetic effect of synbiotic may be accounted to increased expression of GLUT-4 transporter and glucagon like peptide-1 mediated by enhanced production of short-chain fatty acids such as 234 butyrate and propionate, resulting into increased glucose uptake by adipose tissue and 235 muscles ${ }^{23,24}$.

236 Accumulating evidences have demonstrated the role of gut dysbiosis in the etiology and 237 progression of diet induced disorders ${ }^{25}$. The decreased ratio of Bacteroidetes to Firmicutes along 
with decreased abundance of beneficial bacteria i.e. Bifidobacteria spp., Akkermansia spp. along with increased pathobionts i.e. Enterobacteriaceae in HFD animals compared with control is consistent with earlier reports and are considered as "dysbiotic signatures"13,26. This dysbiotic microbiota contributes to metabolic diseases by increasing energy harvest which could be due to downregulation of angiopoietin-like protein 4, a lipoprotein lipase inhibitor inducing host adiposity $^{2}$. Increased energy harvest by dysbiotic microbiota is also supported by fecal microbiota transplantation studies as transfer of the gut microbiota from obese mice even to germ 245 free mice led to an increase in their body weight ${ }^{27}$. Further, increased abundance of LPSproducing Gram negative bacteria along with increased chylomicron formation on HFD intake leads to elevated serum LPS thereby rupturing the gut barrier integrity, causing TLR-4 induced inflammation and endotoxemia ${ }^{6,28}$. Interestingly, in present study, we found that synbiotic exerted its protective effects even in HFD animals via regulating the gut microbiota. The increased Bacteroidetes to Firmicutes ratio along with increased population of Lactobacillus spp., Akkermansia spp., Faecalibacterium spp., Roseburia spp. and decreased abundance of Enterobacteriaceae in feces of synbiotic+HFD animals confirmed the shift from obesogenic to non-obesogenic bacteria and is in agreement with previous study where increased Bacteroidetes to Firmicutes ratio and decreased burden of Enterobacteriaceae was observed on administration of synbiotic i.e. Lactobacillus paracasei HII01 and xylooligosaccharides ${ }^{29}$. Similarly, in another study, Ji et $\mathrm{al}^{30}$ demonstrated increased abundance of Bacteroidetes and Lactobacillus spp. on supplementation of L. sakei CJLS03 to HFD-fed animals. Moreover, Lactobacillus spp., Faecalibacterium spp. and Roseburia spp. are the major contributors of short chain fatty acid production in large intestine, especially butyrate, which has been reported to modulate gut homeostasis by increased hydrolysis of indigestible food, regulating glucose and energy metabolism, promoting satiety and preventing metabolic endotoxemia ${ }^{31,32}$. Furthermore, increased abundance of Akkermansia spp., key producer of acetate and propionate in colon, has been documented to protect against metabolic syndrome by reducing systemic LPS levels and gut permeability in HFD mice, which is possibly associated with the ability of Akkermansia to preserve the mucus layer thickness ${ }^{33,34}$. Therefore, it can be suggested that, increased short chain fatty acid production due to stabilization of gut microbiota on synbiotic supplementation might

267 have altered the adiposity and inflammatory process, influenced the metabolic capability of liver 268 
Metabolic syndrome has also been associated with dyslipidemia, i.e. elevated levels of total cholesterol, triglycerides and LDL-cholesterol, as well as decreased HDL-cholesterol levels, the major risk factors of atherosclerosis leading to cardiovascular diseases. It was observed that synbiotic supplementation to HFD animals positively modulated the serum lipid profile paralleled with reduced hepatic steatosis in histological analysis and increased fecal lipid excretion thereby suggesting that synbiotic administration actually interfered with dietary lipids, making them indigestible and promoting their excretion instead of redistributing to the liver. Similar observations were reported in previous study where scientists have observed improved lipid profile on administration of Lactobacillus plantarum P-8 to hyperlipidemic rats ${ }^{35}$. This may be due to increased bile salt hydrolase activity, cholesterol binding and assimilation by the probiotic cell walls, or physiological actions of the synbiotic metabolites ${ }^{6}$. The improved liver biomarkers (ALT, AST and serum bilirubin) in synbiotic+HFD animals is in concordance with earlier studies where scientists have also observed reduced serum ALT and AST levels upon administration of a probiotic mixture (Lactobacillus and Bifidobacterium) to HFD-fed rats ${ }^{36}$. The observed improved liver plysiology may be due to alleviation of hepatocellular injury induced by lipid deposition and oxidative stress in hepatocytes ${ }^{37}$.

HFD augments oxidative stress due to an imbalance between reactive oxygen species generation and anti-oxidant defense system ${ }^{38}$. Interestingly, in the present study, synbiotic+HFD animals had higher levels of antioxidants (GSH and SOD) and reduced oxidant (MDA) which might be due to quenching of free radicals by probiotic resulting into regulation of host redox status i.e. downregulating enzyme producing reactive oxygen species, chelating metal ions, production of antioxidant metabolites and regulation of gut microbiota ${ }^{39}$. Recently, Li et al. ${ }^{26}$ have also demonstrated the amelioration of liver oxidative stress in HFD-fed mice on oral administration of probiotic mixture of $L$. plantarum strains that led to significantly increased SOD, GSH and reduced MDA levels.

Synbiotic supplementation to HFD animals, downregulated the expression of lipid metabolism regulators, FASN and HSL due to suppressed lipid synthesis, increase $\beta$-oxidation and improved hepatosteatosis and corroborates with earlier studies where selenium enriched probiotics reduced the expression of lipolytic FASN in HFD mice ${ }^{40}$. Similarly, Singh et al. ${ }^{41}$ have also shown decreased HSL expression in cobiotic (IMOs+lycopene) supplemented animals accrediting it to 
anti-inflammatory and antioxidant activity of cobiotic. Further, the liver GLUT-4 mRNA level was enhanced while glucokinase was reduced in synbiotic+HFD animals that may be attributed to the improved insulin resistance, enhanced glycemic control and maintained glucose homeostasis ${ }^{42}$.

HFD elevated the expression of adiposity genes PPAR $\gamma$ and $\mathrm{C} / \mathrm{EBP} \alpha$ in adipose tissue, the main transcription factors of adipocyte differentiation, lipid storage and adipokine signalling ${ }^{43}$. Interestingly, reduced expression of $\operatorname{PPAR} \gamma$ and $\mathrm{C} / \mathrm{EBP} \alpha$ in synbiotic + HFD supplemented animals is suggestive of decreased adipogenesis via limiting the conversion rate of preadipocytes into mature adipocytes. Park et al. ${ }^{44}$ have also reported that L. plantarum Q180 inhibited 3T3-L1 adipocyte differentiation by downregulation of $\mathrm{C} / \mathrm{EBP} \alpha$ and $\operatorname{PPAR} \gamma$, fat absorption and reduction of adipocyte size in diet-induced obese mice. Obesity is often accompanied by resistance to leptin, an important hormone secreted by adipocytes, leading to increased hunger and reduced energy expenditure occurring due to hyperleptinemia ${ }^{45}$. Notably, it was observed that synbiotic supplementation to HFD animals led to decreased leptin mRNA expression indicating improved leptin function and regulation of body fat distribution. Soundharrajan et al. ${ }^{46}$ have also reported that L. plantarum A29 supplementation reduced fat mass and downregulated the expression of leptin gene in adipocytes, resulting in reduced bodyweight of HFD mice.

Adiponectin, an anti-inflammatory adipokine protects against metabolic disorders by regulating glucose levels and promoting fatty acid oxidation, however, its production is inhibited under inflammatory conditions in adipose tissue ${ }^{47}$. It was interesting to observe that animals belonging to synbiotic+ HFD had decreased expression of proinflammatory cytokines, TNF- $\alpha$ and IL-6 and upregulated adiponectin suggesting diminution of inflammation in adipose tissue. Recently, Zeng et al. ${ }^{48}$ have observed that administration of L. pentosus S-PT84 to lipopolysaccharide and HFD fed mice exerted anti-inflammatory effect on adipose tissue by eliminating metabolic endotoxemia-induced macrophage infiltration, restoring the production of adiponectin, and decreased levels of pro-inflammatory mediators.

Systemic endotoxemia, considered as the 'core' of metabolic syndrome is associated with elevated plasma LPS in circulation thereby leading to proinflammatory and oxidant environment $^{49,50}$. Interestingly, reduced serum level of inflammatory markers (LPS, TNF- $\alpha$ and IL-6) in synbiotic+ HFD animals highlighted the anti-inflammatory potential of this intervention 
in alleviating metabolic endotoxemia. Lim et al. ${ }^{21}$ also observed that L. sakei OK67 supplementation antagonized the HFD-induced alterations by inhibiting LPS production, regulating tight junction protein expression and suppressing inflammation. The synbioticmediated alleviation of chronic inflammation could be accredited to decreased number of LPS producing Enterobacteriacea in gut microbiota and downregulation of key signaling pathways i.e. NF- $\mathrm{KB}$ and MAPK by probiotic or its metabolites as observed in our earlier in vitro study, where L. pentosus GSSK2 attenuated LPS-induced inflammation by downregulating MAPK pathway vis-a-vis inhibiting COX-2 $2^{14,51}$.

HFD intake induces an increase in gut permeability by reducing the thickness of mucus layer, impairing the expression of tight junction proteins via LPS-induced activation of TLR-4 pathway $^{25}$. In the present study, mucin gene Muc-2 and tight junction protein claudin were upregulated with reduced expression of TLR-4 in colon suggesting that synbiotic supplementation to HFD animals attenuated mucosal damage and regulated the gut barrier function which is further supported by improved colon morphology with reduced infilteration of immune cells in synbiotic+HFD supplemented animals. Similarly, Mennigen et al. ${ }^{52}$ reported that probiotic mixture VSL\#3 protected the epithelial barrier function by maintaining tight junction protein expression in a murine model of colitis while Castro-Rodríguez ${ }^{53}$ documented reduced systemic inflammation due to decreased TLR-4 expression in Leuconostoc mesenteroides subsp. mesenteroides SD23 supplemented HFD mice.

Based on the present study, synbiotic biointervention, a combination of probiotic L. pentosus GSSK2 and prebiotic IMOs was found to be the most effective and comparable to antiobesity drug orlistat in terms of improved anthropometric parameters, biochemical markers, gene expression and histoarchitecture of adipose tissue, colon and liver. Therefore, the proposed molecular mechanism of modulation of HFD-induced metabolic alterations by synbiotic may be attributed to remodulation of gut microbiota by probiotic as well as prebiotic, that may have led to altered adiposity by remodeling energy metabolism, activation of nutrient sensing pathways, mobilization of fats by regulating the expression of glucose and lipid metabolism genes, reduced lipid absorption due to increased fatty acid oxidation, cholesterol binding and assimilation by probiotic. Moreover, increased short chain fatty acid production due to biofermentation of prebiotic and reduced circulating LPS levels due to decreased pathobionts may have led to 
359 mitigation of chronic inflammation, alleviation of metabolic endotoxemia, oxidative stress and 360 restoration of intestinal barrier function that in turn regulated the glucose homeostasis. Reduced 361 inflammatory adipokines due to alleviation of systemic inflammation might have prevented fat 362 accumulation in liver and adipose tissue hampering the vicious cycle between metabolic and 363 immune responses thereby preventing the progression of metabolic syndrome.

364 Taken together, it is proposed that such novel synbiotic intervention may be employed for 365 combating the growing incidence of metabolic syndrome that could be considered as a promising 366 and alternative live bacteriotherapy for maintaining the immune- metabolic homeostasis.

367 However, due to species and strain specific response of probiotics and entirely different gut 368 microbiota of rodent, the present observations need to be validated clinically.

\section{Methods}

\section{Animals}

371 Male Sprague-Dawley (SD) rats (150-180g) were procured from inbred population of the Central 372 Animal House, Panjab University, Chandigarh, India. Rats were housed in polypropylene cages 373 with a hygienic bed of husk in room with $12 \mathrm{~h}$ light/dark cycle, acclimatized for 7-10 days and 374 given standard pellet diet and water ad libitum.

\section{Ethics declaration}

376 All protocols related to the sampling, care and management of animals were approved by 377 Institutional Animals Ethical Committee (IAEC), Panjab University, Chandigarh and the 378 Committee for the Purpose of Control and Supervision on Experiments on Animals 379 (PU/45/99/CPCSEA/IAEC/2017/27). All experiments were performed in accordance with 380 Institutional guidelines and regulations. The study is reported in accordance with ARRIVE 381 guidelines.

\section{Preparation of HFD}

383 Standard pellet diet (SPD) (6\% calories from fat) was procured from Ashirwad Industries, 384 Chandigarh, India and HFD (60\% calories from fat) was prepared in-house as described 385 previously ${ }^{15}$.

\section{Preparation of dose}


- Probiotic: $18 \mathrm{~h}$ old culture of indigenous probiotic L. pentosus GSSK2 was centrifuged at $4,000 \times g$ for 10 minutes at $4^{\circ} \mathrm{C}$, washed, and suspended in phosphate buffer saline (PBS pH 7.4) to contain $1 \times 10^{9}$ lactobacilli/0.1 $\mathrm{ml}^{15}$.

- Prebiotic: IMOs $\left(1 \mathrm{~g} / \mathrm{kg}\right.$ body weight/ $\left.0.1 \mathrm{~mL} \mathrm{PBS}^{41}\right)$ was used as prebiotic.

- Synbiotic: Probiotic L. pentosus GSSK2 $\left(1 \times 10^{9}\right.$ lactobacilli/0.1 ml) in combination with prebiotic IMOs (1 $\mathrm{g} / \mathrm{kg}$ body weight), was employed as synbiotic.

\section{Experimental design}

Animals were divided into nine groups, each comprising of 6 animals and treated as follows.

- Group I (Control): Animals were fed with SPD for 12 weeks.

- Group II (HFD) : Animals were fed with HFD for 12 weeks.

- Group III (L. pentosus GSSK2): Animals were fed with a single dose of probiotic (1 x $10^{9}$ lactobacilli/0.1mL) daily via orogastric gavage and were given SPD for 12 weeks.

- Group IV (L. pentosus GSSK2+ HFD): Animals were fed orally with a single dose of probiotic $\left(1 \times 10^{9}\right.$ lactobacilli/0.1mL) daily along with HFD for 12 weeks.

- Group V (IMOs): Animals belonging to this group were fed orally with a single dose of IMOs (1g/kg body weight) daily along with SPD for 12 weeks.

- Group VI (IMOs+ HFD): Animals were fed orally with a single dose of IMOs ( $1 \mathrm{~g} / \mathrm{kg}$ body weight/ $0.1 \mathrm{~mL}$ ) daily along with HFD for 12 weeks.

- Group VII (Synbiotic): Animals were fed orally with a single dose of both probiotic (1 x $10^{9}$ lactobacilli/0.1 mL) and IMOs (1g/kg body weight) daily along with SPD for 12 weeks.

- Group VIII (Synbiotic+ HFD): Animals were fed orally with a single dose of both probiotic $\left(1 \times 10^{9}\right.$ lactobacilli/0.1mL) and IMOs (1g/kg body weight) daily along with HFD for 12 weeks.

- Group IX (Orlistat+ HFD): Animals were fed orally with a single dose of orlistat (10mg/kg body weight/ $0.1 \mathrm{~mL}$ PBS) daily along with HFD for 12 weeks.

\section{Follow up of animals}

Body weight and lactic acid bacteria (LAB) count were monitored once a week, throughout the experiment. A day before sacrificing the animals, fasting blood glucose level was monitored, oral glucose tolerance test (OGTT) was performed and feces of animals were collected, for estimation of fecal lipids and analysis of gut bacterial abundance. Animals were sacrificed after 12 weeks of 
respective treatments by injecting ketamine hydrochloride $(80 \mathrm{mg} / \mathrm{kg})$ intraperitoneally followed by cervical dislocation. Blood was drawn through retro-orbital bleeding for estimation of serum biochemical parameters. Liver, adipose tissue (epididymal and retroperitoneal) and colon were collected for analysis of oxidants and antioxidants, histopathological alterations and molecular markers.

\section{Evaluation of anthropometric parameters and adiposity markers}

Body weight of animals was recorded weekly on ordinary balance (SD-300, S.D fine chemicals Ltd, Chandigarh, India) while abdominal circumference was measured at the beginning and end of the study using ordinary measuring tape ${ }^{15}$. Lee's Index was calculated as cube root of body weight $(\mathrm{g}) /$ naso-anal length $(\mathrm{cm})$, Body mass index (BMI) was monitored as body weight $(\mathrm{g}) /$ length ${ }^{2}\left(\mathrm{~cm}^{2}\right)$ at the end of experiment ${ }^{13}$.

Feed intake of animals was recorded twice a month and was calculated by subtracting the amount of residual food in each cage from the weighed amount of food provided on previous day (g/day) and represented as average feed intake (g/day/ rat) by dividing the feed intake by total number of animals per cage ${ }^{15}$. Post sacrifice, liver and adipose tissue were weighed using ordinary balance.

\section{Blood glucose and OGTT}

The fasting blood glucose levels of animals were recorded weekly via tail snip method using glucometer (Freestyle Optium Glucometer, Abbott diabetes care Ltd., Oxon, UK). For OGTT, animals were fasted for $6 \mathrm{~h}$ and blood glucose level was measured before and after oral administration of D-glucose $(2 \mathrm{~g} / \mathrm{kg})$ at an interval of $15,30,60,90$, and 120 minutes respectively ${ }^{15}$. Area under the concentration-time curve (AUC) was calculated using GraphPad PRISM 5 software.

\section{Fecal LAB count and fecal lipids}

To assess the effect of synbiotic supplementation on LAB in the colon, freshly voided fecal material (0.5 g/animal) was collected weekly from each group, homogenized in normal saline, serially diluted and plated on MRS agar. The plates were incubated at $37^{\circ} \mathrm{C}$ for $48 \mathrm{~h}$ and colony forming units (CFU) were recorded ${ }^{11}$.

Fecal lipids were extracted using phase separation based method by Folch et al. ${ }^{54}$ followed by estimation of total lipids by method of Fringes and Dunn ${ }^{55}$. Briefly, $200 \mathrm{mg}$ dry feces was taken in a centrifuge tube and $3 \mathrm{~mL}$ of chloroform-methanol mixture $(2: 1, \mathrm{v} / \mathrm{v})$ was added, vortexed 
449 for 1 minute and centrifuged at 3,000 g for 10 minutes. The chloroform phase containing lipid 450 fraction was collected in a fresh tube and completely dried followed by estimation of total lipids.

451 Selected gut bacterial abundance

452 Bacterial DNA was isolated using QIAmp® DNA stool mini kit (Qiagen, Hilden, Germany) 453 from $180 \mathrm{mg}$ of fecal sample, as per manufacturer's instruction. DNA quantification was 454 performed using Infinite ${ }^{\circledR}$ M200 Pro NanoQuant (Tecan). DNA extracted as above was 455 subjected to qPCR to quantify the abundance of Lactobacillus spp., Bifidobacterium spp., 456 Roseburia spp., Akkermansia spp., Faecalibacterium spp., Ruminococcus spp., Prevotella spp., 457 using genus-specific primers and that of Enterobacteriaceae, Bacteroidetes and Firmicutes using 458 phylum-specific primers taking total bacteria as an internal control. q-PCR conditions and primer 459 details are given in supplementary material (Supplementary Table 1). Data was analyzed using 460 the $\Delta \Delta \mathrm{Ct}$ method and values expressed as fold change relative to the control group ${ }^{13}$.

461 Analysis of serum biochemical parameters

462 Blood was collected retro-orbitally and serum was prepared to estimate liver function test 463 [Bilirubin, aspartate transaminase (AST) and alanine transaminase (ALT)] and lipid profile 464 [Total cholesterol, triglycerides, high-density lipoprotein (HDL) cholesterol and low-density 465 lipoprotein (LDL) cholesterol] using autoanalyser, Sysmex XP-100. Serum lipopolysaccharide 466 (LPS), TNF- $\alpha$ and IL-6 were quantified using commercially available ELISA kits (BT 467 Laboratory, Zhejiang, China) as per manufacturers' instructions.

468 Assessment of oxidant and antioxidant level

469 Tissue homogenates of colon, liver and adipose tissue samples were prepared in 0.15 M PBS (pH 470 7.2) using potter Elvehjem homogenizer. Post mitochondrial supernatant (PMS) was prepared 471 by cold centrifuging tissues homogenates at $16,000 \times \mathrm{g}$ for 10 minutes and supernatant was 472 labeled as PMS. Protein concentration in tissue homogenate and PMS was measured using 473 standard method of Lowry et al. ${ }^{56}$.

474 The amount of malondialdehyde (MDA), a measure of lipid peroxidation, was assayed in 475 homogenates as per Wills ${ }^{57}$ and results were expressed as nanomoles of MDA per milligram of 476 protein. Superoxide dismutase (SOD) activity was assayed in PMS of tissue homogenates 477 according to the method of $\mathrm{Kono}^{58}$ and expressed as units of SOD per milligram of protein, 478 where $1 \mathrm{U}$ activity is defined as the amount of SOD required to inhibit the rate of Nitroblue 479 tetrazolium reduction by 50\%. Reduced glutathione (GSH) levels were estimated in tissue 
480 homogenates as per Ellman ${ }^{59}$, absorbance was measured at $412 \mathrm{~nm}$ and results were expressed as $481 \mu$ mole of $\mathrm{GSH} / \mathrm{mg}$ of protein.

482 Gene expression analysis

483 q-PCR based gene expression analysis was done for fatty acid synthase (FASN), hormone sensitive lipase (HSL), glucokinase, GLUT-4, TNF- $\alpha$ and IL-6 in liver; CCAAT/ enhancerbinding protein alpha $(\mathrm{C} / \mathrm{EBP} \alpha)$, peroxisome proliferators-activated receptor gamma (PPAR- $\gamma$ ),

486 leptin, adiponectin, TNF- $\alpha$ and IL-6 in adipose tissue; claudin, CDX-2, Muc2 and TLR-4 in 487 colon. Total RNA was extracted using Trizol (Sigma Aldrich, USA). $1 \mu \mathrm{g}$ of RNA sample was used for c-DNA synthesis using commercially available kit (Biorad iscript kit 1708891) as per the kit's instructions. Relative expression of different genes was determined by qPCR using SYBR $®$ based dye (Biorad C1000 Touch Real-Time PCR machine). q-PCR conditions and primer details are given in supplementary material (Supplementary Table 2). Data was analyzed using the $\Delta \Delta \mathrm{Ct}$ method and values were expressed as fold change relative to control group.

493 GAPDH was used as internal reference gene to normalize the expression of target genes.

494 Histological analysis

495 A part of distal colon, liver and adipose tissue were fixed immediately in $10 \%$ buffered formalin, 496 processed, stained with hematoxylin and eosin, and examined for histological alterations using 497 light microscope. The mean adipocyte sizes in adipose tissue sections (minimum 2 animals per 498 group) were estimated in 10-12 images (40X objective), using Image $\mathrm{J}$ software ${ }^{60}$.

\section{Statistical analysis}

500 Results were expressed as mean \pm standard deviation (SD). The inter group variation was 501 assessed by one-way analysis of variance (ANOVA) followed by Tukey's post Hoc Test using 502 PRISM software (5.0). The statistical significance was defined as $\mathrm{p}$ and calculated at $\mathrm{p}<0.05$.

\section{References}

505 1. Saklayen, M. G. The Global Epidemic of the Metabolic Syndrome. Curr Hypertens Rep $506 \quad 20,018-0812(2018)$.

507 2. Arora, T. \& Bäckhed, F. The gut microbiota and metabolic disease: current understanding $508 \quad$ and future perspectives. J Intern Med 280, 339-349 (2016). 
3. Wang, P.-X., Deng, X.-R., Zhang, C.-H. \& Yuan, H.-J. Gut microbiota and metabolic syndrome. Chin Med J (Engl) 133, 808-816, doi:10.1097/cm9.0000000000000696 (2020).

4. Dabke, K., Hendrick, G. \& Devkota, S. The gut microbiome and metabolic syndrome. $J$ Clin Invest 129, 4050-4057 (2019).

5. Garg, A. Acquired and inherited lipodystrophies. N Engl J Med 350, 1220-1234 (2004).

6. Xavier-Santos, D., Bedani, R., Lima, E. D. \& Saad, S. M. I. Impact of probiotics and prebiotics targeting metabolic syndrome. $J$ Funct Foods 64, 103666, doi:https://doi.org/10.1016/j.jff.2019.103666 (2020).

7. George Kerry, R. et al. Benefaction of probiotics for human health: A review. J Food Drug Anal 26, 927-939, doi:https://doi.org/10.1016/j.jfda.2018.01.002 (2018).

8. Chandel, D., Sharma, M., Chawla, V., Sachdeva, N. \& Shukla, G. Isolation, characterization and identification of antigenotoxic and anticancerous indigenous probiotics and their prophylactic potential in experimental colon carcinogenesis. Sci Rep 9, 14769, doi:10.1038/s41598-019-51361-z (2019).

9. Gibson, G. R. et al. Expert consensus document: The International Scientific Association for Probiotics and Prebiotics (ISAPP) consensus statement on the definition and scope of prebiotics. Nat Rev Gastroenterol Hepatol 14, 491-502, doi:10.1038/nrgastro.2017.75 (2017).

10. Davani-Davari, D. et al. Prebiotics: Definition, Types, Sources, Mechanisms, and Clinical Applications. Foods 8, 92, doi:10.3390/foods8030092 (2019).

11. Verma, A. \& Shukla, G. Synbiotic (Lactobacillus rhamnosus+Lactobacillus acidophilus+inulin) attenuates oxidative stress and colonic damage in 1,2 dimethylhydrazine dihydrochloride-induced colon carcinogenesis in Sprague-Dawley rats: a long-term study. Eur J Cancer Prev 23, 550-559 (2014).

12. Ke, X. et al. Synbiotic-driven improvement of metabolic disturbances is associated with changes in the gut microbiome in diet-induced obese mice. Mol Metab 22, 96-109 (2019).

13. Singh, D. P. et al. Isomalto-oligosaccharides, a prebiotic, functionally augment green tea effects against high fat diet-induced metabolic alterations via preventing gut dysbacteriosis in mice. Pharmacol Res 123, 103-113 (2017). 
539 14. Khanna, S., Bishnoi, M., Kondepudi, K. K. \& Shukla, G. Isolation, characterization and 540 anti-inflammatory mechanism of probiotics in lipopolysaccharide-stimulated RAW 264.7 macrophages. World J Microbiol Biotechnol 36, $74 \quad$ (2020). https://doi.org/10.1007/s11274-020-02852-z>.

54315 Khanna, S., Walia, S., Kondepudi, K. K. \& Shukla, G. Administration of indigenous 544 probiotics modulate high-fat diet-induced metabolic syndrome in Sprague Dawley rats. Antonie Van Leeuwenhoek 113, 1345-1359 (2020).

16. Kang, J. G. \& Park, C.-Y. Anti-Obesity Drugs: A Review about Their Effects and Safety. Diabetes Metab J 36, 13-25, doi:10.4093/dmj.2012.36.1.13 (2012).

17. Yadav, H., Lee, J. H., Lloyd, J., Walter, P. \& Rane, S. G. Beneficial metabolic effects of 549 a probiotic via butyrate-induced GLP-1 hormone secretion. J Biol Chem 288, 2508825097 (2013).

19. Esposito, E. et al. Probiotics reduce the inflammatory response induced by a high-fat diet

18. Martinez-Guryn, K. et al. Small Intestine Microbiota Regulate Host Digestive and Absorptive Adaptive Responses to Dietary Lipids. Cell Host Microbe 23, 458-469 (2018).

23. Nakamura, Y. K. \& Omaye, S. T. Metabolic diseases and pro- and prebiotics: Mechanistic insights. Nutr Metab 9, 1743-7075 (2012).

24. Balakumar, M. et al. Improvement in glucose tolerance and insulin sensitivity by 568 probiotic strains of Indian gut origin in high-fat diet-fed C57BL/6J mice. Eur J Nutr 57, 279-295 (2018). 
25. Li, X., Watanabe, K. \& Kimura, I. Gut Microbiota Dysbiosis Drives and Implies Novel Therapeutic Strategies for Diabetes Mellitus and Related Metabolic Diseases. Front Immunol 8, doi:10.3389/fimmu.2017.01882 (2017).

26. Li, H. et al. Probiotic Mixture of Lactobacillus plantarum Strains Improves Lipid Metabolism and Gut Microbiota Structure in High Fat Diet-Fed Mice. Front Microbiol 11, doi:10.3389/fmicb.2020.00512 (2020).

27. Turnbaugh, P. J. et al. An obesity-associated gut microbiome with increased capacity for energy harvest. Nature 444, 1027-1031 (2006).

28. Kim, K. A., Gu, W., Lee, I. A., Joh, E. H. \& Kim, D. H. High fat diet-induced gut microbiota exacerbates inflammation and obesity in mice via the TLR4 signaling pathway. PLoS One 7, 16 (2012).

29. Thiennimitr, P. et al. Lactobacillus paracasei HII01, xylooligosaccharides, and synbiotics reduce gut disturbance in obese rats. Nutrition 54, doi:10.1016/j.nut.2018.03.005 (2018).

30. Ji, Y. et al. Amelioration of obesity-related biomarkers by Lactobacillus sakei CJLS03 in a high-fat diet-induced obese murine model. Sci Rep 9, 019-43092 (2019).

31. Tamanai-Shacoori, Z. et al. Roseburia spp.: a marker of health? Future Microbiol 12, 157-170 (2017).

32. Vallianou, N., Stratigou, T., Christodoulatos, G. S. \& Dalamaga, M. Understanding the Role of the Gut Microbiome and Microbial Metabolites in Obesity and ObesityAssociated Metabolic Disorders: Current Evidence and Perspectives. Curr Obes Rep 8, 317-332, doi:10.1007/s13679-019-00352-2 (2019).

33. Everard, A. et al. Cross-talk between Akkermansia muciniphila and intestinal epithelium controls diet-induced obesity. Proc Natl Acad Sci U S A 110, 9066-9071 (2013).

34. Xu, Y. et al. Function of Akkermansia muciniphila in Obesity: Interactions With Lipid Metabolism, Immune Response and Gut Systems. Front Microbiol 11, doi:10.3389/fmicb.2020.00219 (2020).

35. Bao, Y. et al. Effect of Lactobacillus plantarum P-8 on lipid metabolism in hyperlipidemic rat model. European J Lipid Sci Technol 114, 1230-1236, doi:https://doi.org/10.1002/ejlt.201100393 (2012).

36. Liang, Y. et al. Probiotic mixture of Lactobacillus and Bifidobacterium alleviates systemic adiposity and inflammation in non-alcoholic fatty liver disease rats through 
Gpr109a and the commensal metabolite butyrate. Inflammopharmacology 26, 1051-1055 (2018).

37. Gratz, S. W., Mykkanen, H. \& El-Nezami, H. S. Probiotics and gut health: a special focus on liver diseases. World J Gastroenterol 16, 403-410, doi:10.3748/wjg.v16.i4.403 (2010).

38. Tan, B. L. \& Norhaizan, M. E. Effect of High-Fat Diets on Oxidative Stress, Cellular Inflammatory Response and Cognitive Function. Nutrients 11, 2579, doi:10.3390/nu11112579 (2019).

39. Wang, Y. et al. Antioxidant Properties of Probiotic Bacteria. Nutrients 9, 521, doi:10.3390/nu9050521 (2017).

40. Nido, S. A. et al. Effects of Selenium-Enriched Probiotics on Lipid Metabolism, Antioxidative Status, Histopathological Lesions, and Related Gene Expression in Mice Fed a High-Fat Diet. Biol Trace Elem Res 171, 399-409 (2016).

41. Singh, D. P. et al. A novel cobiotic-based preventive approach against high-fat dietinduced adiposity, nonalcoholic fatty liver and gut derangement in mice. Int $J$ Obes 40, 487-496 (2016).

42. Shulman, G. I. Ectopic fat in insulin resistance, dyslipidemia, and cardiometabolic disease. N Engl J Med 371, 1131-1141 (2014).

43. Moseti, D., Regassa, A. \& Kim, W. K. Molecular Regulation of Adipogenesis and Potential Anti-Adipogenic Bioactive Molecules. Int J Mol Sci 17 (2016).

44. Park, S.-Y., Kim, S. \& Lim, S.-D. The Inhibitory Effect of L. plantarum Q180 on Adipocyte Differentiation in 3T3-L1 and Reduction of Adipocyte Size in Mice Fed Highfat Diet. Korean J Food Sci Anim Resour 38, 99-109, doi:10.5851/kosfa.2018.38.1.99 (2018).

45. Izquierdo, A. G., Crujeiras, A. B., Casanueva, F. F. \& Carreira, M. C. Leptin, Obesity, and Leptin Resistance: Where Are We 25 Years Later? Nutrients 11, 2704, doi:10.3390/nu11112704 (2019).

46. Soundharrajan, I. et al. Positive metabolic effects of selected probiotic bacteria on dietinduced obesity in mice are associated with improvement of dysbiotic gut microbiota. The FASEB Journal 34, 12289-12307, doi:https://doi.org/10.1096/fj.202000971R (2020). 
47. Achari, A. E. \& Jain, S. K. Adiponectin, a Therapeutic Target for Obesity, Diabetes, and Endothelial Dysfunction. Int J Mol Sci 18 (2017).

48. Zeng, Y., Zhang, H., Tsao, R. \& Mine, Y. Lactobacillus pentosus S-PT84 Prevents LowGrade Chronic Inflammation-Associated Metabolic Disorders in a Lipopolysaccharide and High-Fat Diet C57/BL6J Mouse Model. J Agric Food Chem 68, 4374-4386 (2020).

49. Boutagy, N. E., McMillan, R. P., Frisard, M. I. \& Hulver, M. W. Metabolic endotoxemia with obesity: Is it real and is it relevant? Biochimie 124, 11-20 (2016).

50. Cani, P. D. et al. Metabolic endotoxemia initiates obesity and insulin resistance. Diabetes 56, 1761-1772 (2007).

51. Dai, C. et al. VSL\#3 probiotics exerts the anti-inflammatory activity via PI3k/Akt and NF-אB pathway in rat model of DSS-induced colitis. Mol Cell Biochem 374, 1-11, doi:10.1007/s11010-012-1488-3 (2013).

52. Mennigen, R. et al. Probiotic mixture VSL\#3 protects the epithelial barrier by maintaining tight junction protein expression and preventing apoptosis in a murine model of colitis. Am J Physiol Gastrointest Liver Physiol 296, 12 (2009).

53. Castro-Rodríguez, D. C. et al. Leuconostoc mesenteroides subsp. mesenteroides SD23 Prevents Metabolic Dysfunction Associated with High-Fat Diet-Induced Obesity in Male Mice. Probiotics Antimicrob Proteins 12, 505-516 (2020).

54. Folch, J., Lees, M. \& Sloane Stanley, G. H. A simple method for the isolation and purification of total lipides from animal tissues. J Biol Chem 226, 497-509 (1957).

55. Frings, C. S. \& Dunn, R. T. A colorimetric method for determination of total serum lipids based on the sulfo-phospho-vanillin reaction. Am J Clin Pathol 53, 89-91 (1970).

56. Lowry, O. H., Rosebrough, N. J., Farr, A. \& Randall, R. J. Protein measurement with the folin Phenol Reagent. J. biol. Chemistry 193, 256-275, doi:10.1016/s00219258(19)52451-6 (1951).

57. Wills, E. D. Mechanisms of lipid peroxide formation in animal tissues. Biochem J 99, 667-676 (1966).

58. Kono, Y. Generation of superoxide radical during autoxidation of hydroxylamine and an assay for superoxide dismutase. Arch Biochem Biophys 186, 189-195 (1978).

59. Ellman, G. L. Tissue sulfhydryl groups. Arch Biochem Biophys 82, 70-77 (1959). 
660

661

662

663

664

665

666

667

668

669

670

671

672

673

674

675

676

677

678

679

680

681

682

683

684

685

686

60. Sarma, S. M. et al. Finger millet arabinoxylan protects mice from high-fat diet induced lipid derangements, inflammation, endotoxemia and gut bacterial dysbiosis. Int J Biol Macromol 106, 994-1003 (2018).

\section{Acknowledgements}

The financial support provided by the Indian Council of Medical Research, India (3/1/3 JRF2016/HRD-51) for carrying the present study; Panjab University, Chandigarh and National Agrifood Biotechnology Institute, Mohali for providing research facilities are highly acknowledged.

\section{Author Contributions}

S.K: Conducted experiments, compiled and analyzed data, and wrote manuscript; K.K.K: Supervised the study, helped in analyzing and interpreting data M.B.: Helped in designing diet and gene expression experiment; G.S.: Conceived, helped in analyzing, interpreting data and editing the manuscript along with overall supervision of the project.

Conflicts of interest: There is no conflict of interest among the authors.

\section{Figure Legend}

Figure 1: Anthropometric parameters and adiposity markers in different groups of animals: (a) Body weight; (b) Weight gain; (c) Liver and adipose tissue weight; (d) BMI $\left(\mathrm{g} / \mathrm{cm}^{2}\right)$ and Lee's index (e) Change in abdominal circumference; (f) Feed intake; Values are Mean $\pm \mathrm{SD},{ }^{\#} \mathrm{p}<0.05$ versus control, ${ }^{*} \mathrm{p}<0.05$ versus HFD.

Figure 2: Effect of probiotic, prebiotic and synbiotic supplementation in animals belonging to various groups on: (a) Fasting blood glucose; (b) OGTT (c) AUC of OGTT; (d) Fecal lactic acid bacteria count ( $\left.\log _{10} \mathrm{CFU} / \mathrm{ml}\right)$; (e) Fecal lipid excretion. Values are Mean $\pm \mathrm{SD},{ }^{\#}<0.05$ versus control, $* \mathrm{p}<0.05$ versus HFD.

Figure 3: Relative bacterial abundance of different genera in animals belonging to different groups: (a) Bacteroidetes; (b) Firmicutes; (c) Bacteroidetes: Firmicutes; (d) Lactobacillus, Bifidobacteria, Akkermansia, Faecalibacterium, Roseburia, Ruminococcus, Prevotella, Enterobacteriaceae, by real-time PCR. Values are Mean $\pm \mathrm{SD},{ }^{\#} \mathrm{p}<0.05$ versus control, ${ }^{*} \mathrm{p}<0.05$ versus HFD. 
Figure 4: Relative gene expression of: (a) lipid metabolism genes, inflammatory markers, glucose metabolism genes in liver; (b) adiposity genes in adipose tissue; (c) gut integrity genes in colon of animals belonging to various groups by real-time PCR. Values are Mean $\pm \mathrm{SD},{ }^{\#} \mathrm{p}<0.05$ versus control, ${ }^{*} \mathrm{p}<0.05$ versus HFD.

Figure 5: Photomicrograph of adipose tissue showing: (a) normal histomorphology with uniform, spherical adipocytes in control animals; (b) hypertrophied adipocytes in HFD; (c, e \& g) normal histoarchitecture of adipocytes in L. pentosus, IMOs and synbiotic animals; (d, f, h \& i) reduced adiposity in L. pentosus + HFD, IMOs + HFD, synbiotic + HFD and orlistat + HFD animals ( $\mathrm{H} \& \mathrm{E}$ staining; scale bar: $50 \mu \mathrm{m}, 400 \mathrm{X}$ ); (j) mean adipocyte size in animals belonging to different groups.

Figure 6: Photomicrograph of liver of animals belonging to different groups showing: (a) Normal histomorphology with polyhedral hepatocytes having large, rounded vesicular nuclei in control; (b) severe hepatic steatosis and ballooning degeneration of hepatocytes in HFD; (c, e \& g) normal histoarchitectureof hepatocytes in L. pentosus, IMOs and synbiotic animals; (f) ballooned hepatocytes with vacuolated nuclei in IMOs + HFD animals; (d, h \& i) reduced hepatic steatosis in L. pentosus + HFD, synbiotic + HFD and orlistat + HFD animals (H \& E staining; arrows indicate ballooned hepatocytes; scale bar: $50 \mu \mathrm{m}, 400 \mathrm{X})$.

Figure 7: Photomicrograph of colon of animals belonging to different groups depicting: (a) normal histoarchitecture showing mucosa, submucosa, muscularis propria and serosa in control; (b) severely damaged mucosa with infilteration of lymphocytes and plasma cells in HFD; (c, e \& g) normal histomorphology of colon in L. pentosus, IMOs and synbiotic animals; (f) inflammation in IMOs + HFD animals; (d, h \& i) reduced infilteration of immune cells with intact mucosa in L. pentosus + HFD, synbiotic + HFD and orlistat + HFD animals (H \& E staining; arrows indicate infilteration of inflammatory cells; scale bar: $100 \mu \mathrm{m}, 100 \mathrm{X})$. 
717 Table 1: Serum biochemical parameters (lipid profile, liver function test, LPS, TNF- $\alpha$ and IL-6)

718 of animals belonging to different groups

\begin{tabular}{|c|c|c|c|c|c|c|c|c|c|}
\hline \multirow[b]{2}{*}{ Parameter } & \multicolumn{9}{|c|}{ Groups } \\
\hline & Control & HFD & $\begin{array}{l}\text { L. } \\
\text { pentosus }\end{array}$ & $\begin{array}{l}\text { L. } \\
\text { pentosus } \\
\text { +HFD }\end{array}$ & IMOs & $\begin{array}{l}\text { IMOs+ } \\
\text { HFD }\end{array}$ & Synbiotic & $\begin{array}{l}\text { Synbiotic+ } \\
\text { HFD }\end{array}$ & $\begin{array}{l}\text { Orlistat+ } \\
\text { HFD }\end{array}$ \\
\hline $\begin{array}{l}\text { Total } \\
\text { cholesterol } \\
(\mathrm{mg} / \mathrm{dl})\end{array}$ & $70.2 \pm 9.5$ & $110.8^{\#} \pm 9.9$ & $75.6 \pm 7.9$ & $98.4^{\#} \pm 8.3$ & $78 \pm 9.0$ & $93^{\#} \pm 10.4$ & $71.4 \pm 9.2$ & $79.8^{*} \pm 9.6$ & $82.6^{*} \pm 7.0$ \\
\hline $\begin{array}{l}\text { Triglycerides } \\
\text { (mg/dl) }\end{array}$ & $82 \pm 5.6$ & $118.4^{\#} \pm 11.7$ & $76.8 \pm 6.2$ & $95.6^{*} \pm 5.2$ & $79.8 \pm 7.9$ & $98.2^{*} \pm 9.6$ & $85 \pm 11.5$ & $91^{*} \pm 9.2$ & $89.2^{*} \pm 10.2$ \\
\hline $\begin{array}{l}\text { HDL } \\
\text { cholesterol } \\
(\mathrm{mg} / \mathrm{dl})\end{array}$ & $47.7 \pm 4.4$ & $32.1^{\#} \pm 4.3$ & $43.2 \pm 5.3$ & $39.8 \pm 3.6$ & $43 \pm 4.2$ & $38 \pm 5.1$ & $45.2 \pm 4.1$ & $45^{*} \pm 7.1$ & $45.4^{*} \pm 2.1$ \\
\hline $\begin{array}{l}\text { LDL } \\
\text { cholesterol } \\
(\mathrm{mg} / \mathrm{dl})\end{array}$ & $23.06 \pm 3.4$ & $40.72^{\#} \pm 2.7$ & $28 \pm 4.2$ & $26.4^{*} \pm 6.5$ & $26.8 \pm 5.2$ & $41.4^{\#} \pm 7.7$ & $22.2 \pm 8.2$ & $25.3^{*} \pm 4.3$ & $27.2^{*} \pm 5.5$ \\
\hline $\begin{array}{l}\text { SGOT (AST) } \\
\text { (IU/L) }\end{array}$ & $95.04 \pm 9.8$ & $160.8^{\#} \pm 9.9$ & $100.6 \pm 8.7$ & $132.2^{*} \pm 8.0$ & $104.4 \pm 7.1$ & $147^{\#} \pm 8.7$ & $97.8 \pm 4.3$ & $106.6^{*} \pm 5.4$ & $103.6^{*} \pm 8.1$ \\
\hline $\begin{array}{l}\text { SGPT (ALT) } \\
\text { (IU/L) }\end{array}$ & $62 \pm 9.6$ & $123.6^{\#} \pm 11.6$ & $66.4 \pm 6.6$ & $80.8^{\# *} \pm 7.9$ & $79 \pm 8.3$ & $115^{\#} \pm 10.2$ & $62 \pm 6.4$ & $73^{*} \pm 5.4$ & $74.4^{*} \pm 6.1$ \\
\hline $\begin{array}{l}\text { Serum } \\
\text { bilirubin } \\
\text { (mg/dl) }\end{array}$ & $0.48 \pm 0.02$ & $1.08^{\#} \pm 0.03$ & $0.46 \pm 0.02$ & $0.66^{*} \pm 0.05$ & $0.57 \pm 0.04$ & $0.72^{*} \pm 0.01$ & $0.52 \pm 0.03$ & $0.58^{*} \pm 0.06$ & $0.70^{*} \pm 0.04$ \\
\hline $\begin{array}{l}\text { Serum LPS } \\
(\mathrm{EU} / \mathrm{L})\end{array}$ & $24.8 \pm 3.02$ & $108.5^{\#} \pm 16.3$ & $20.6 \pm 1.7$ & $47.6^{*} \pm 6.9$ & $27.3 \pm 8.4$ & $79.1^{\#} \pm 9.01$ & $28.2 \pm 4.3$ & $38.9^{*} \pm 6.06$ & $42.4^{*} \pm 8.04$ \\
\hline $\begin{array}{l}\text { Serum } \\
\text { TNF-a } \\
(\mathbf{p g} / \mathrm{ml})\end{array}$ & $2.3 \pm 0.76$ & $52.5^{\#} \pm 8.23$ & $2.9 \pm 0.59$ & $30.3^{*} \pm 3.11$ & $4.2 \pm 0.78$ & $41.1^{\#} \pm 9.01$ & $3.6 \pm 0.93$ & $21.3^{*} \pm 1.42$ & $14.7^{*} \pm 0.94$ \\
\hline $\begin{array}{l}\text { Serum IL-6 } \\
(\mathrm{ng} / \mathrm{L})\end{array}$ & $1.9 \pm 0.12$ & $9.2^{\#} \pm 0.73$ & $2.1 \pm 0.51$ & $4.3^{*} \pm 0.95$ & $2.2 \pm 0.14$ & $4.5^{*} \pm 0.52$ & $1.9 \pm 0.15$ & $3.4^{*} \pm 0.17$ & $2.8^{*} \pm 0.24$ \\
\hline
\end{tabular}

719 Values are Mean \pm SD, ${ }^{\#} \mathrm{p}<0.05$ versus control, ${ }^{*} \mathrm{p}<0.05$ versus HFD. 
723 Table 2: Oxidant and antioxidant levels in animals belonging to different groups

\begin{tabular}{|c|c|c|c|c|c|c|c|c|c|}
\hline \multirow{2}{*}{ Groups } & \multicolumn{3}{|c|}{$\begin{array}{c}\text { MDA } \\
(\mathrm{nM} / \mathrm{mg} \text { protein })\end{array}$} & \multicolumn{3}{|c|}{$\begin{array}{c}\text { GSH } \\
\text { (nM/mg protein) }\end{array}$} & \multicolumn{3}{|c|}{$\begin{array}{c}\text { SOD } \\
\text { (U/mg protein) }\end{array}$} \\
\hline & $\begin{array}{l}\text { Adipose } \\
\text { tissue }\end{array}$ & Colon & Liver & $\begin{array}{l}\text { Adipose } \\
\text { tissue }\end{array}$ & Colon & Liver & $\begin{array}{l}\text { Adipose } \\
\text { tissue }\end{array}$ & Colon & Liver \\
\hline Control & $46.6 \pm 5.6$ & $52.2 \pm 4.3$ & $58.3 \pm 7.1$ & $3.54 \pm 0.2$ & $2.72 \pm 0.6$ & $1.44 \pm 0.1$ & $3.12 \pm 0.2$ & $1.16 \pm 0.1$ & $1.25 \pm 0.2$ \\
\hline HFD & $77.1^{\#} \pm 7.8$ & $89.5^{\#} \pm 8.6$ & $91.2^{\#} \pm 4.3$ & $0.84^{\#} \pm 0.3$ & $0.45^{\#} \pm 0.1$ & $0.42^{\#} \pm 0.3$ & $0.71^{\#} \pm 0.1$ & $0.49^{\#} \pm 0.1$ & $0.36^{\#} \pm 0.1$ \\
\hline L. pentosus & $42.2 \pm 3.2$ & $57^{*} \pm 3.6$ & $52.2 \pm 5.5$ & $4.1 \pm 0.31$ & $2.51 \pm 0.3$ & $1.32 \pm 0.6$ & $3.01 \pm 0.1$ & $1.21 \pm 0.3$ & $0.98 \pm 0.1$ \\
\hline $\begin{array}{l}\text { L. pentosus } \\
\text { +HFD }\end{array}$ & $65.2^{*} \pm 5.4$ & $73.8 \pm 4.3$ & $63.8^{*} \pm 4.3$ & $3.09 \pm 0.1$ & $2.03^{*} \pm 0.7$ & $0.99^{*} \pm 0.4$ & $2.6 \pm 0.3$ & $1.01^{*} \pm 0.4$ & $0.66 \pm 0.1$ \\
\hline IMOs & $50.4 \pm 6.5$ & $54.2^{*} \pm 4.6$ & $61.2 \pm 5.9$ & $3.5 \pm 0.3$ & $2.10 \pm 0.3$ & $1.03 \pm 0.5$ & $3.13 \pm 0.4$ & $1.23 \pm 0.2$ & $1.04 \pm 0.3$ \\
\hline $\begin{array}{c}\text { IMOs+ } \\
\text { HFD }\end{array}$ & $71.2 \pm 4.4$ & $83.4 \pm 5.3$ & $88.3 \pm 3.7$ & $1.23 \pm 0.9$ & $0.99 \pm 0.4$ & $0.87 \pm 0.4$ & $1.45 \pm 0.1$ & $0.33 \pm 0.3$ & $0.78 \pm 0.2$ \\
\hline Synbiotic & $48.2 \pm 6.3$ & $50.2 \pm 4.4$ & $60.6 \pm 2.9$ & $3.61 \pm 0.8$ & $2.54 \pm 0.9$ & $1.11 \pm 0.2$ & $2.98 \pm 0.3$ & $1.03 \pm 0.7$ & $1.34 \pm 0.8$ \\
\hline $\begin{array}{c}\text { Synbiotic+ } \\
\text { HFD }\end{array}$ & $55.5^{*} \pm 7.3$ & $59.9 * \pm 6.4$ & $61.2^{*} \pm 6.3$ & $3.2^{*} \pm 0.7$ & $1.99^{*} \pm 0.7$ & $0.98^{*} \pm 0.3$ & $2.12^{*} \pm 0.6$ & $1.05^{*} \pm 0.2$ & $0.99^{*} \pm 0.3$ \\
\hline $\begin{array}{c}\text { Orlistat+ } \\
\text { HFD }\end{array}$ & $60.6^{*} \pm 4.3$ & $55.5 \pm 4.3$ & $65.5^{*} \pm 4.4$ & $3.01 \pm 0.6$ & $1.84^{*} \pm 0.1$ & $0.52 \pm 0.1$ & $2.44^{*} \pm 0.4$ & $1.09^{*} \pm 0.3$ & $0.54 \pm 0.5$ \\
\hline
\end{tabular}

724 Values are Mean \pm SD, ${ }^{*} \mathrm{p}<0.05$ versus control, ${ }^{*} \mathrm{p}<0.05$ versus HFD.

725

726 

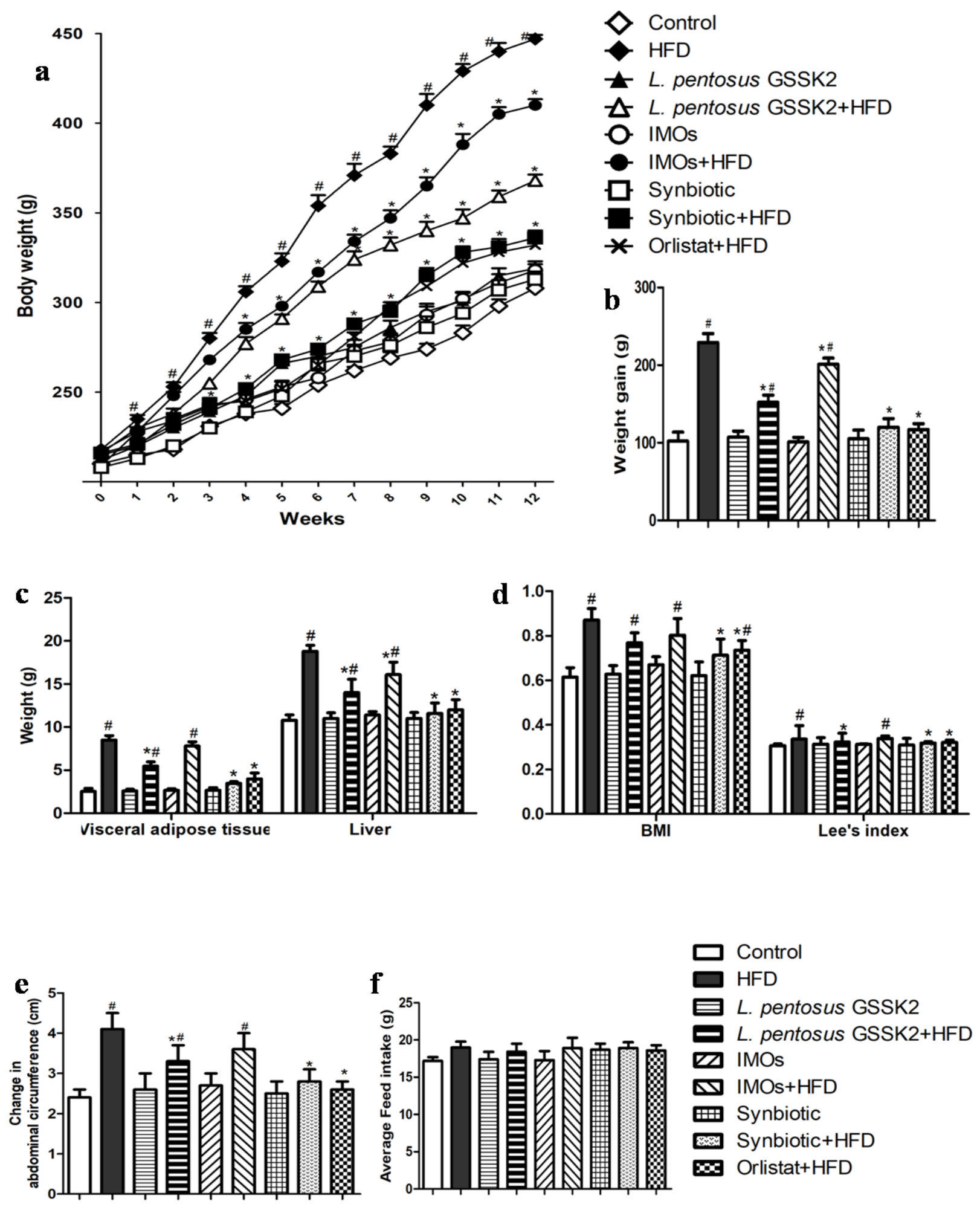


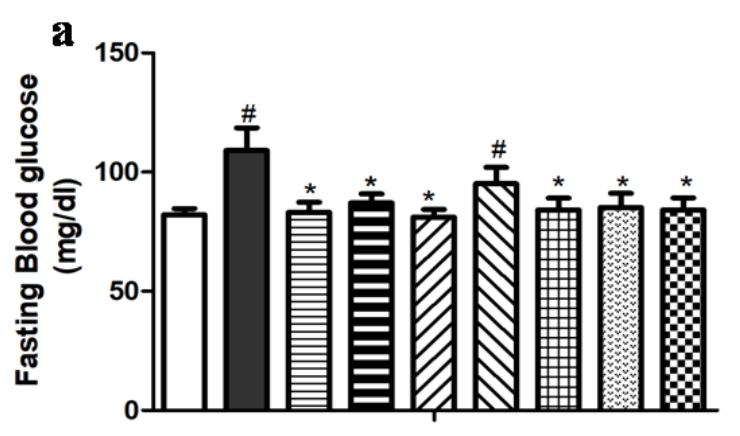

$\square$ Control
$\square$ HFD
$\square$ L.pentosus GSSK2
$\square$ L.pentosus GSSK2+HFD
$\square$ IMOs
$\square$ IMOs+HFD
Synbiotic
Synbiotic+HFD
$\mathbf{0}$ Orlistat+HFD
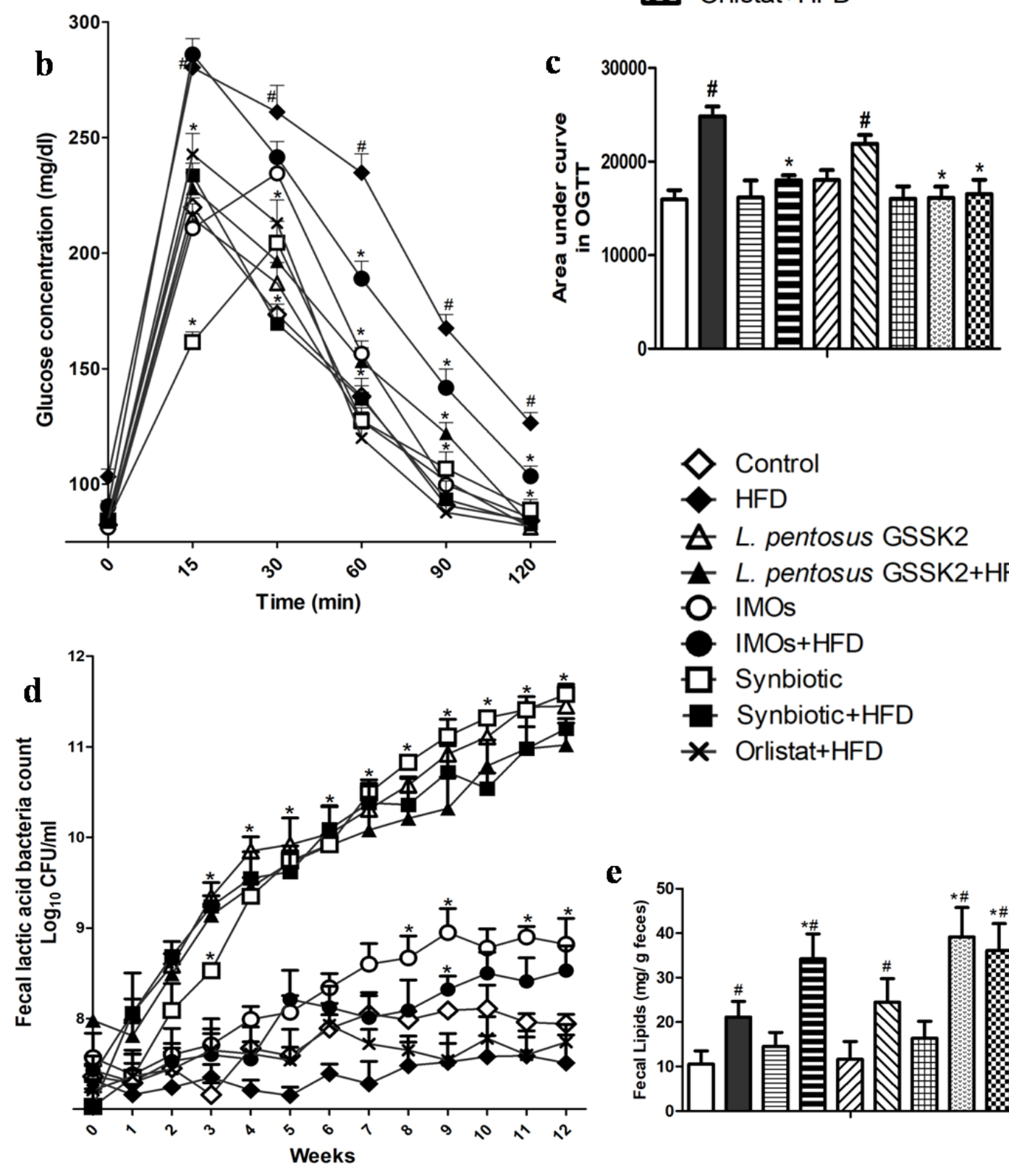
$\diamond$ Control
$\checkmark$ HFD
$\triangle$ L. pentosus GSSK2
^ L. pentosus GSSK2+HFD
O IMOs
- IMOs+HFD
$\square$ Synbiotic
- Synbiotic+HFD
* Orlistat+HFD


a

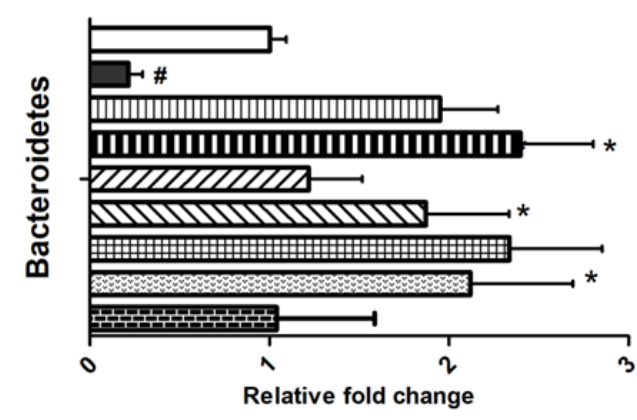

b

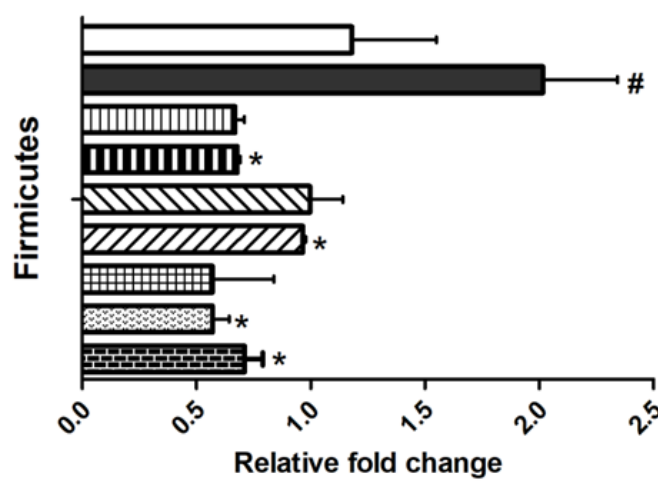

d

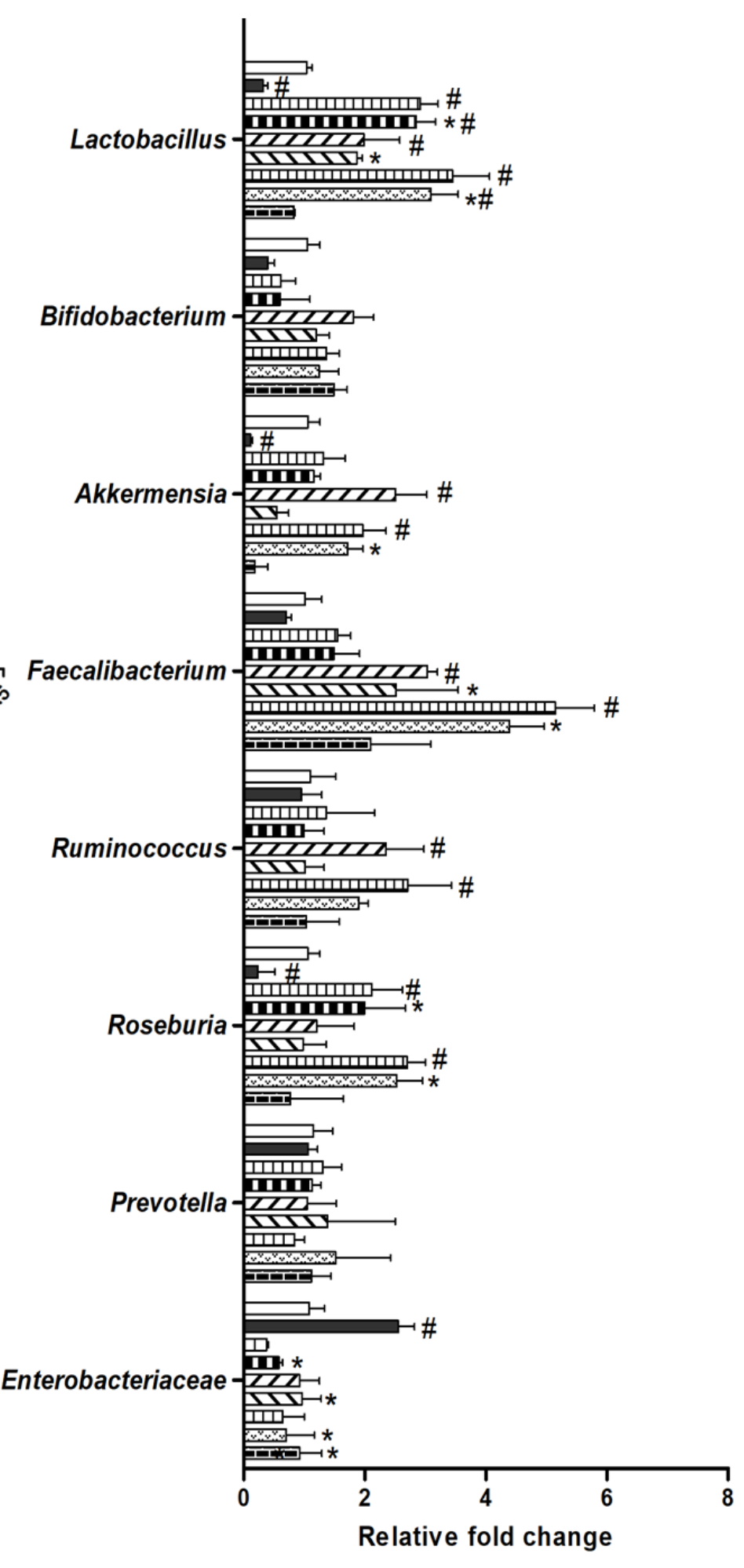

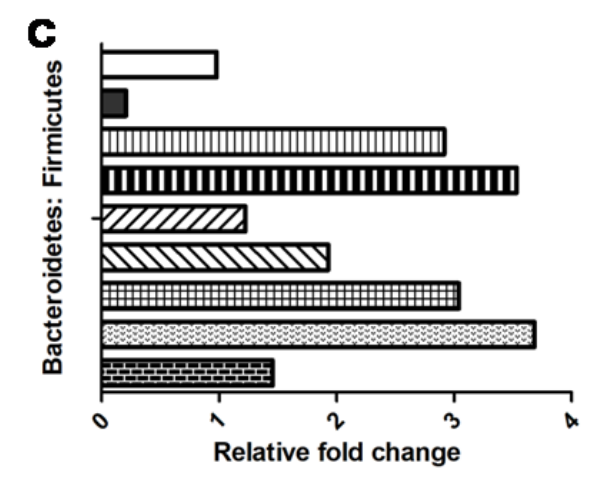

$\square$ Control

$\square$ HFD

س L. pentosus GSSK2

III L. pentosus GSSK2+HFD

ZD IMOs

$\Delta \nabla$ IMOs + HFD

世罗 Synbiotic

Synbiotic + HFD

Orlistat+HFD

Figure 3 
a
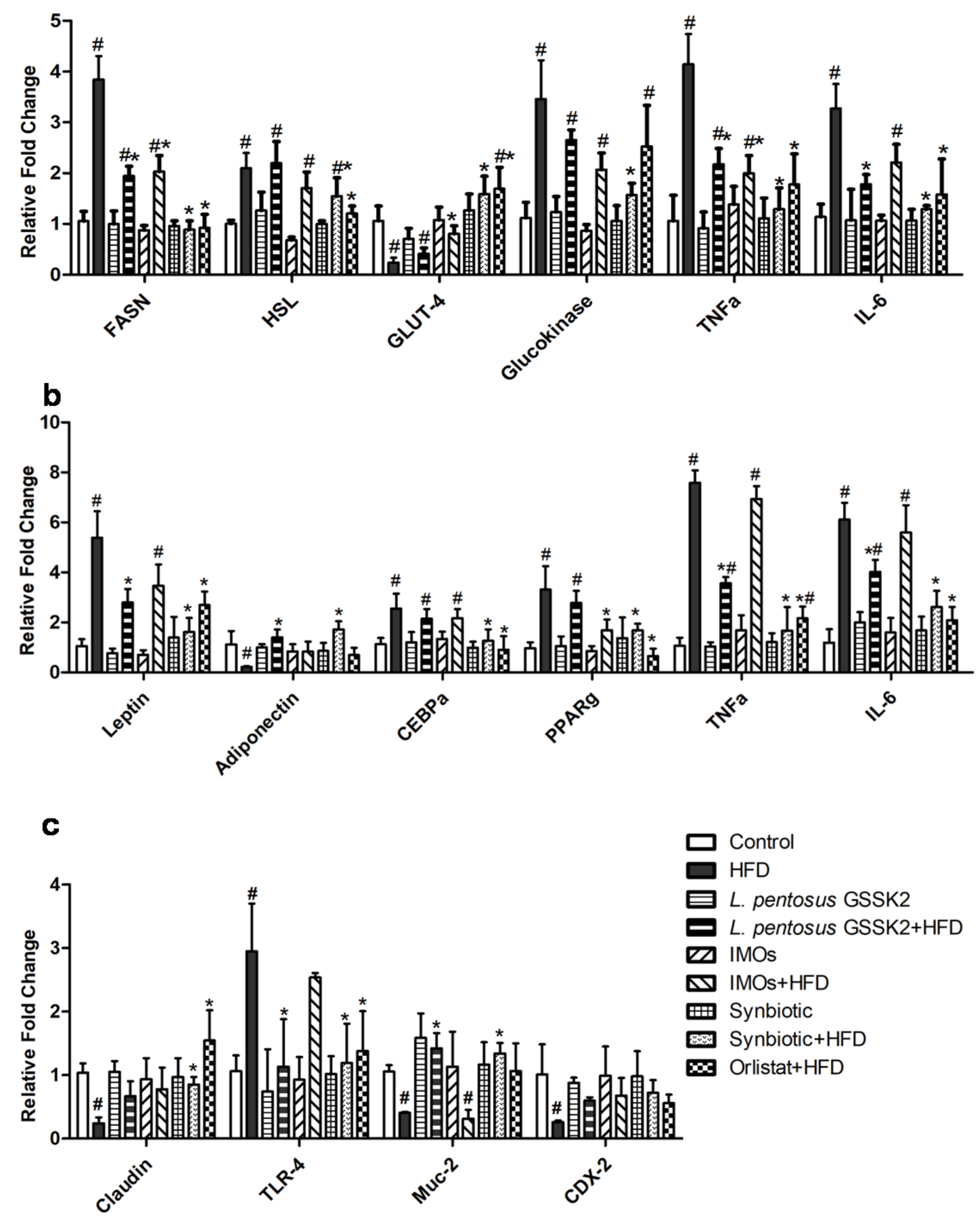

Figure 4 

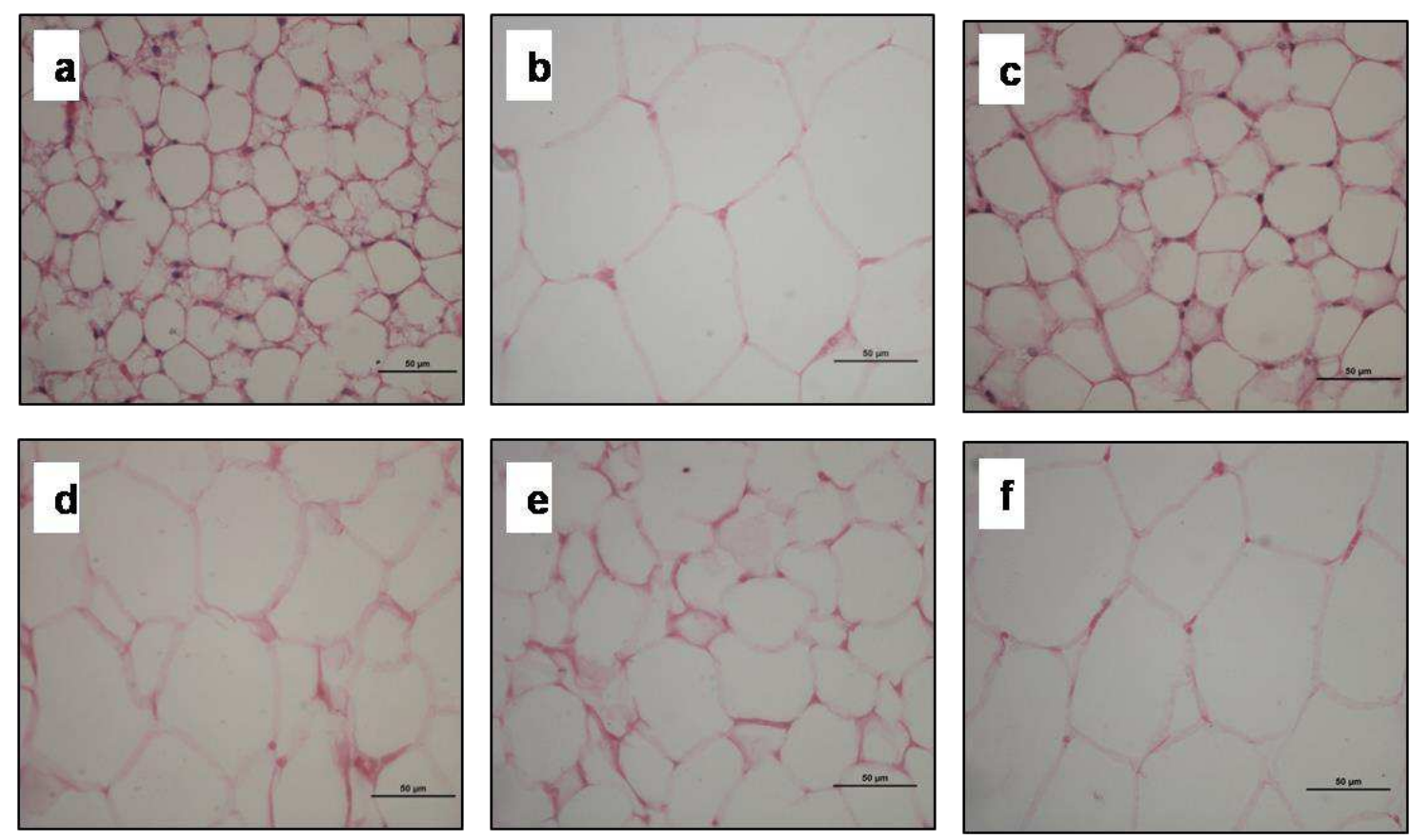

736
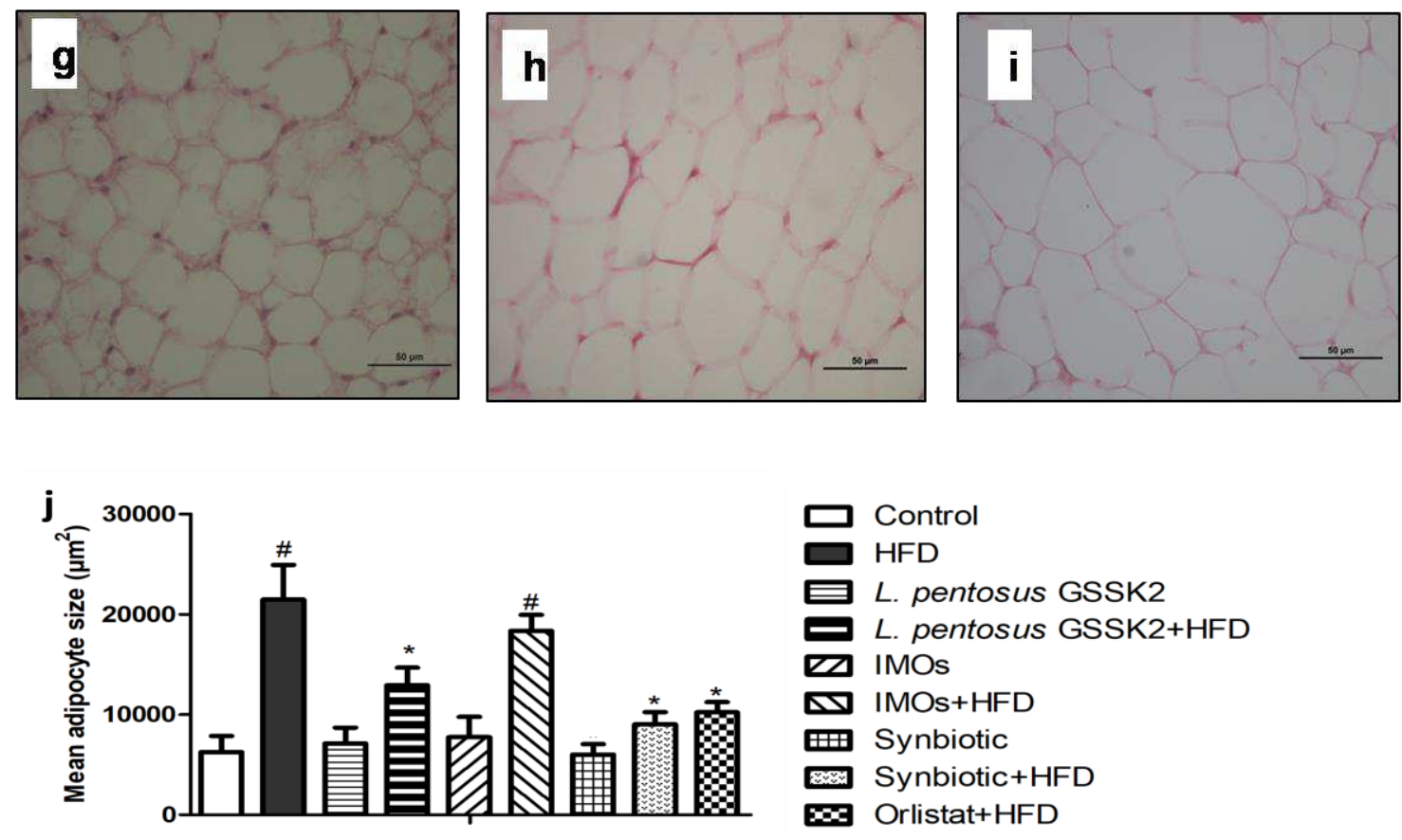

Figure 5 

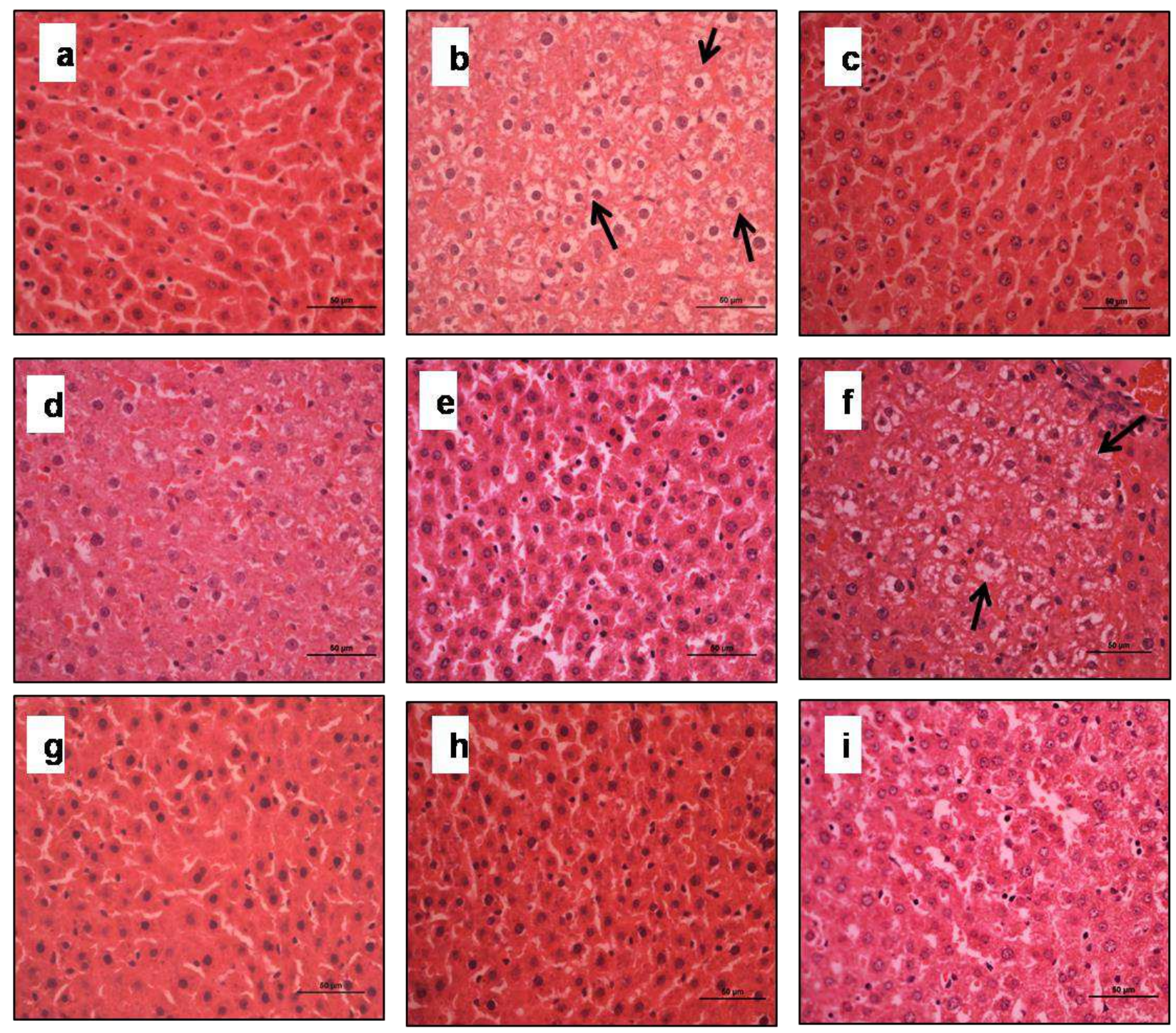

741

Figure 6

742

743

744 

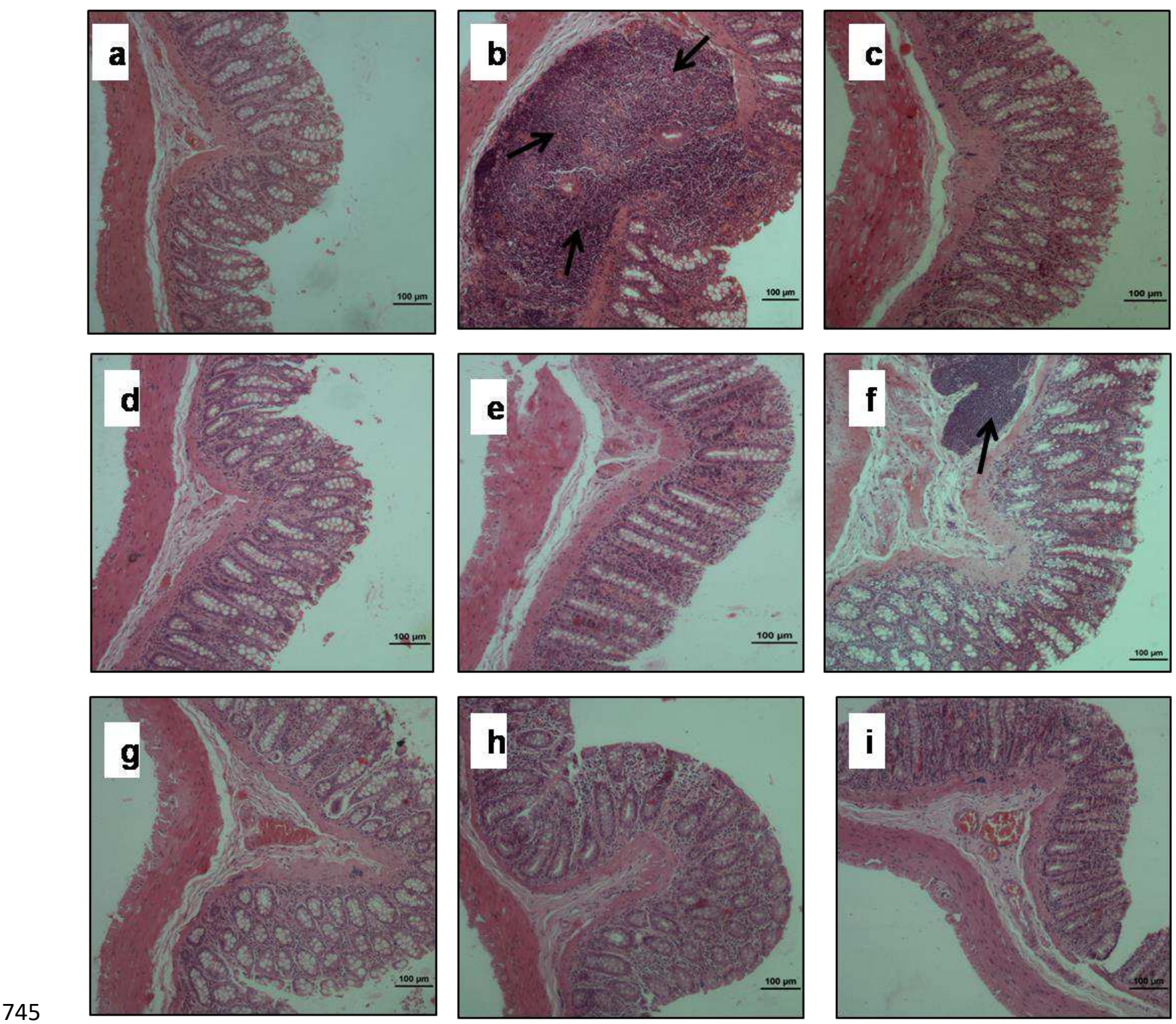

Figure 7 


\section{Figures}
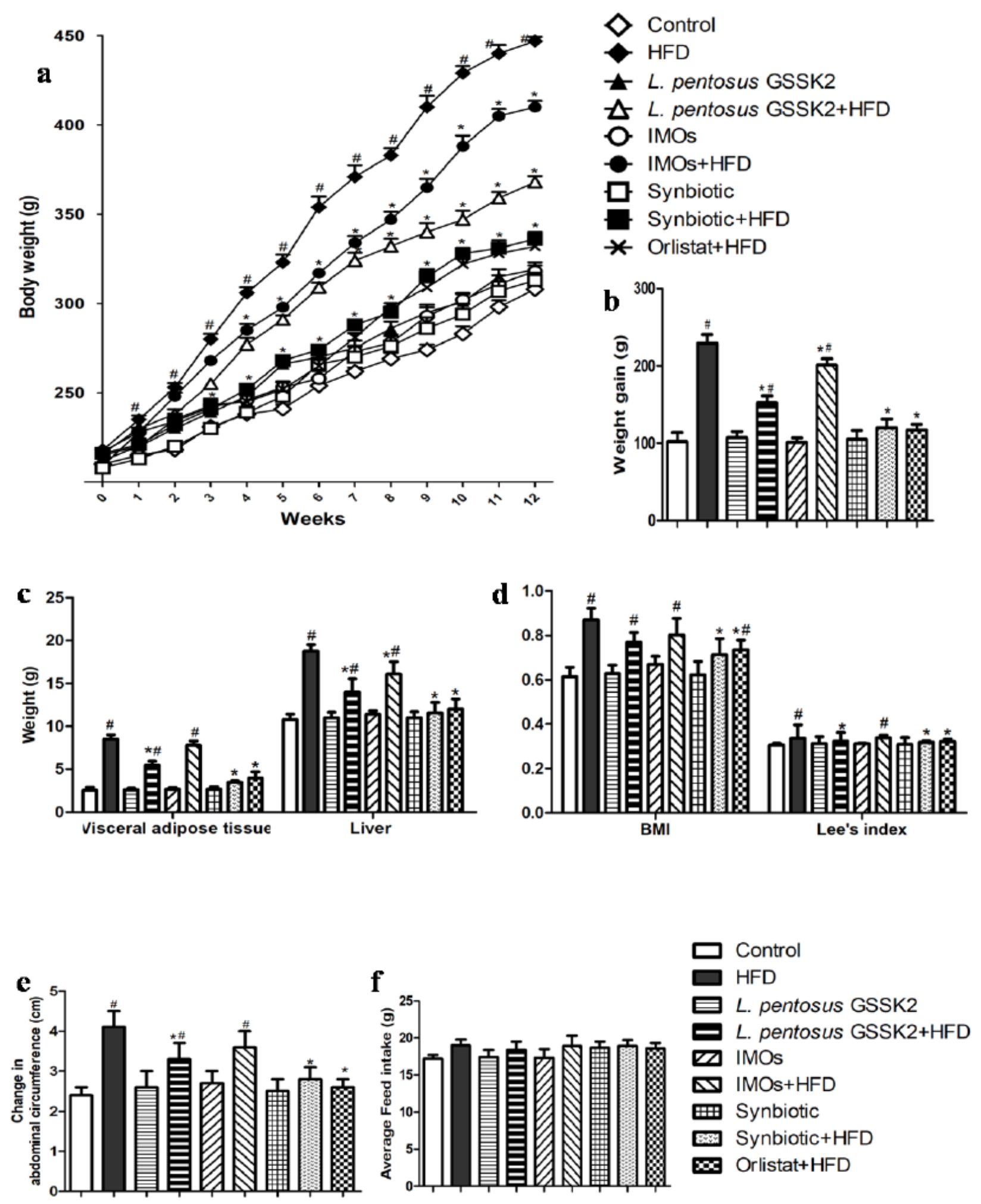

Figure 1

Anthropometric parameters and adiposity markers in different groups of animals: (a) Body weight; (b) Weight gain; (c) Liver and adipose tissue weight; (d) BMI (g/cm2) and Lee's index (e) Change in 
abdominal circumference; (f) Feed intake; Values are Mean $\pm S D$, $\# p<0.05$ versus control, ${ }^{*} p<0.05$ versus HFD.
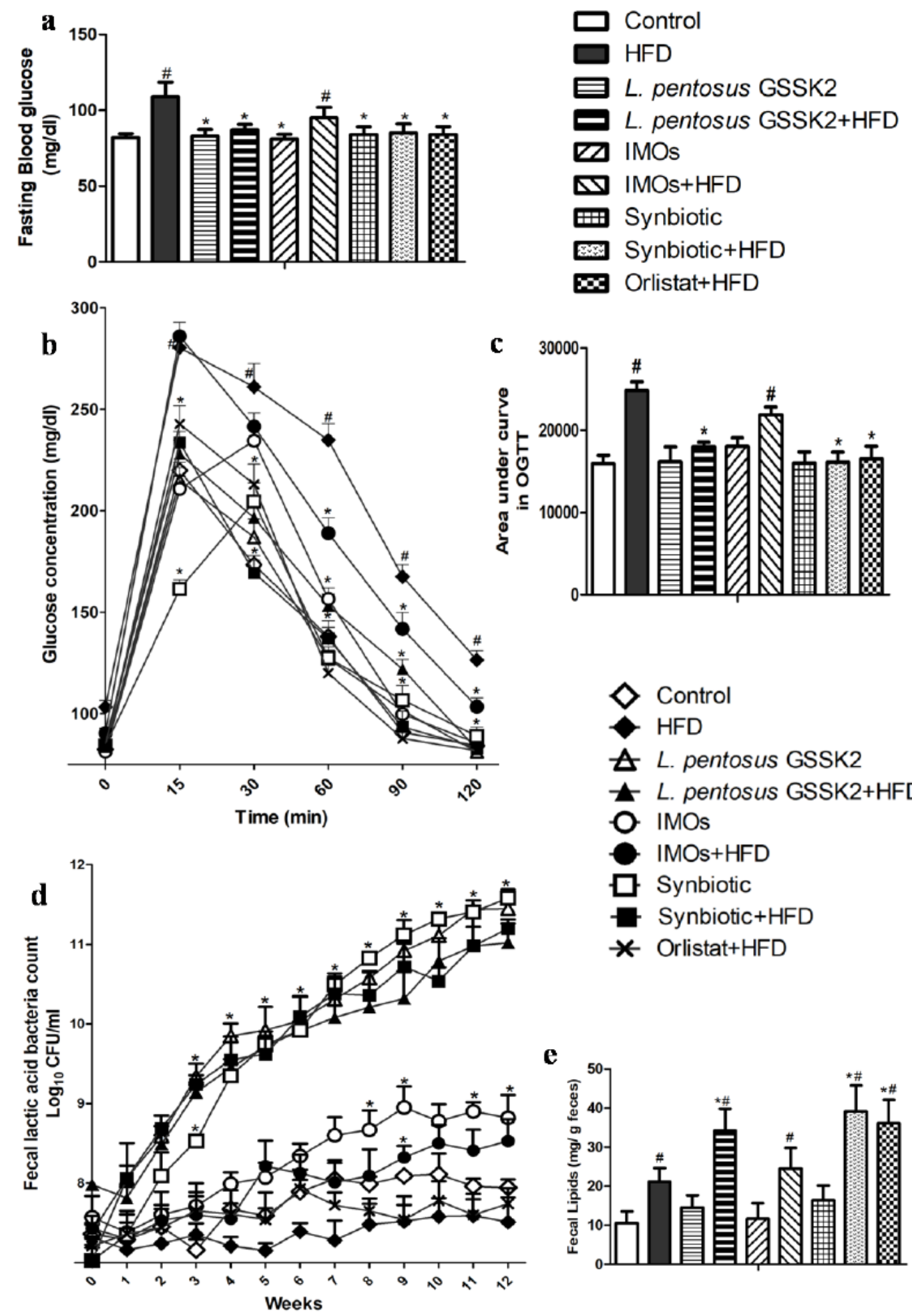

Figure 2

Effect of probiotic, prebiotic and synbiotic supplementation in animals belonging to various groups on: (a) Fasting blood glucose; (b) OGTT (c) AUC of OGTT; (d) Fecal lactic acid bacteria count (Log10 $\mathrm{CFU} / \mathrm{ml}$ ); (e) Fecal lipid excretion. Values are Mean $\pm S \mathrm{SD}, \# p<0.05$ versus control, ${ }^{*} p<0.05$ versus HFD. 
a

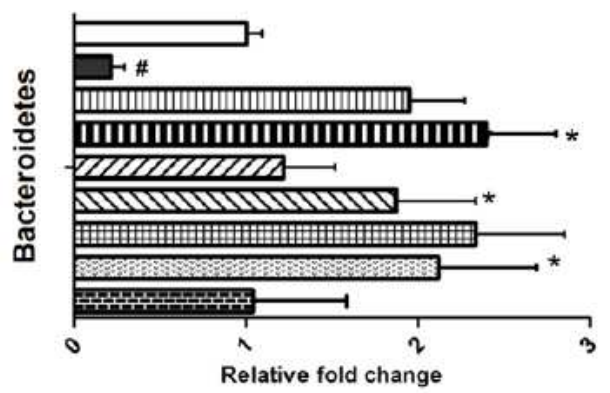

b
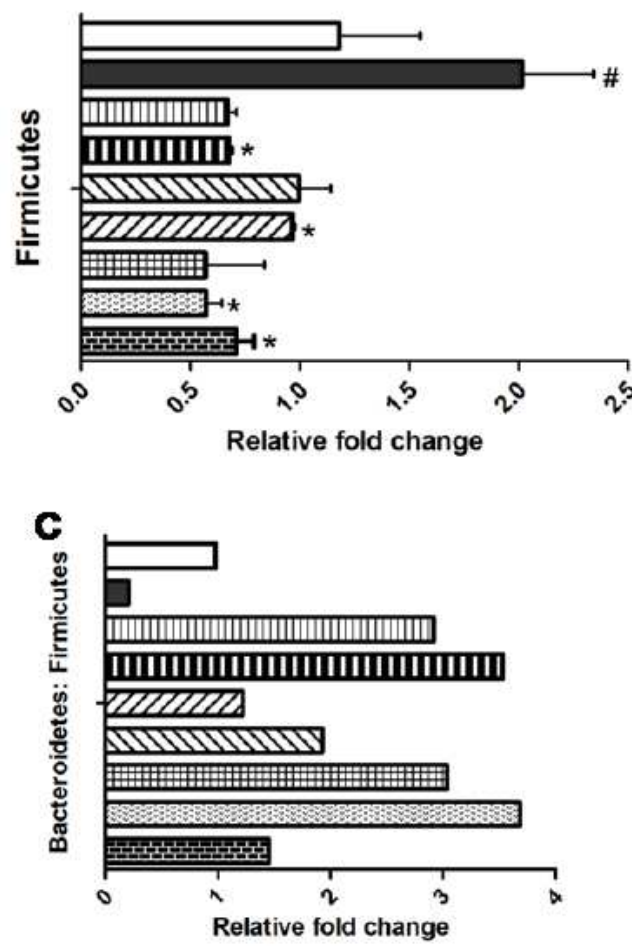

$\square$ Control

HFD

سI L. pentosus GSSK2

II L. pentosus GSSK2+HFD

DI IMOs

$\triangle \mathrm{IMOS}+\mathrm{HFD}$

Synbiotic

Eynbiotic + HFD

Orlistat+HFD d

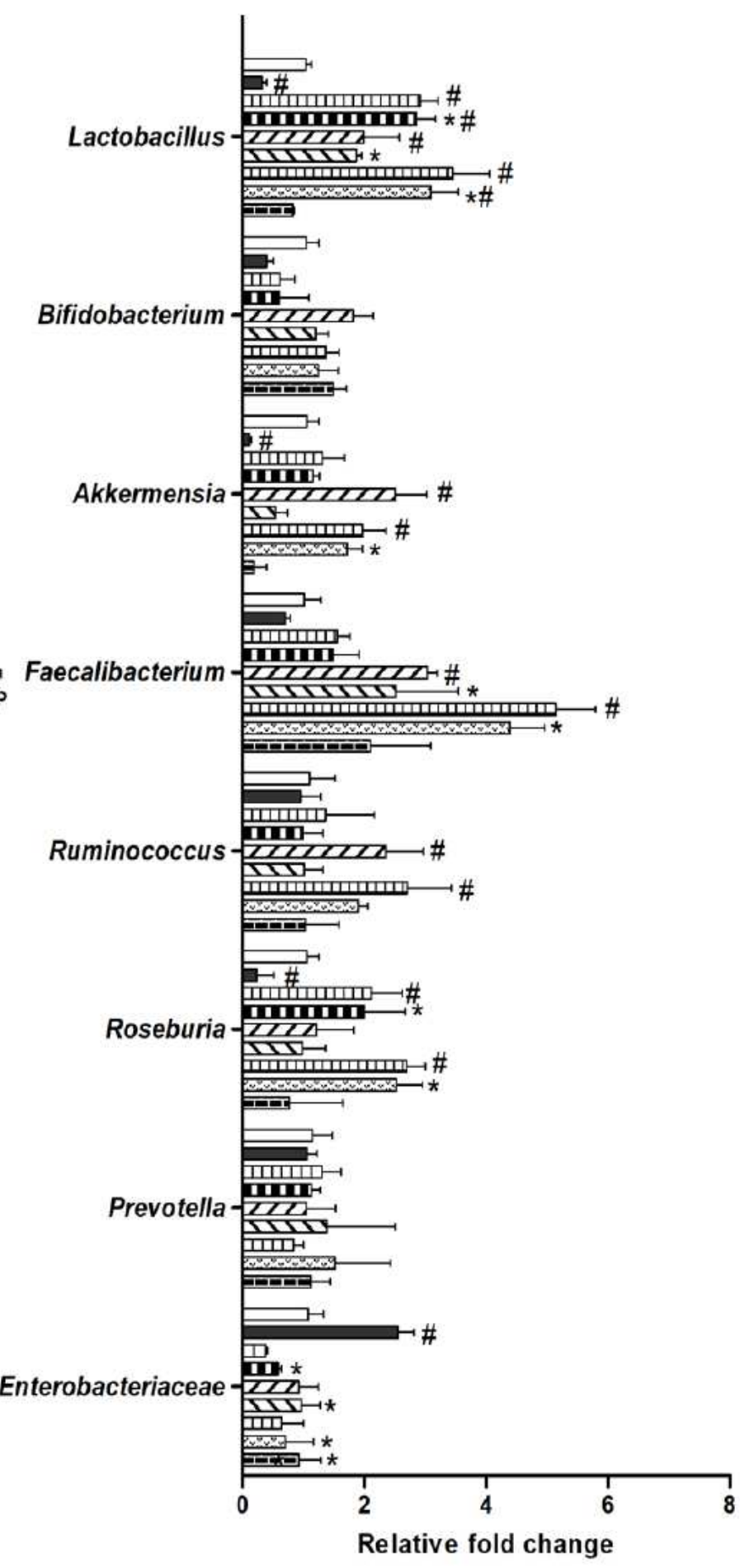

\section{Figure 3}

Relative bacterial abundance of different genera in animals belonging to different groups: (a) Bacteroidetes; (b) Firmicutes; (c) Bacteroidetes: Firmicutes; (d) Lactobacillus, Bifidobacteria, Akkermansia, Faecalibacterium, Roseburia, Ruminococcus, Prevotella, Enterobacteriaceae, by real-time PCR. Values are Mean $\pm S D, \# p<0.05$ versus control, $* p<0.05$ versus HFD. 

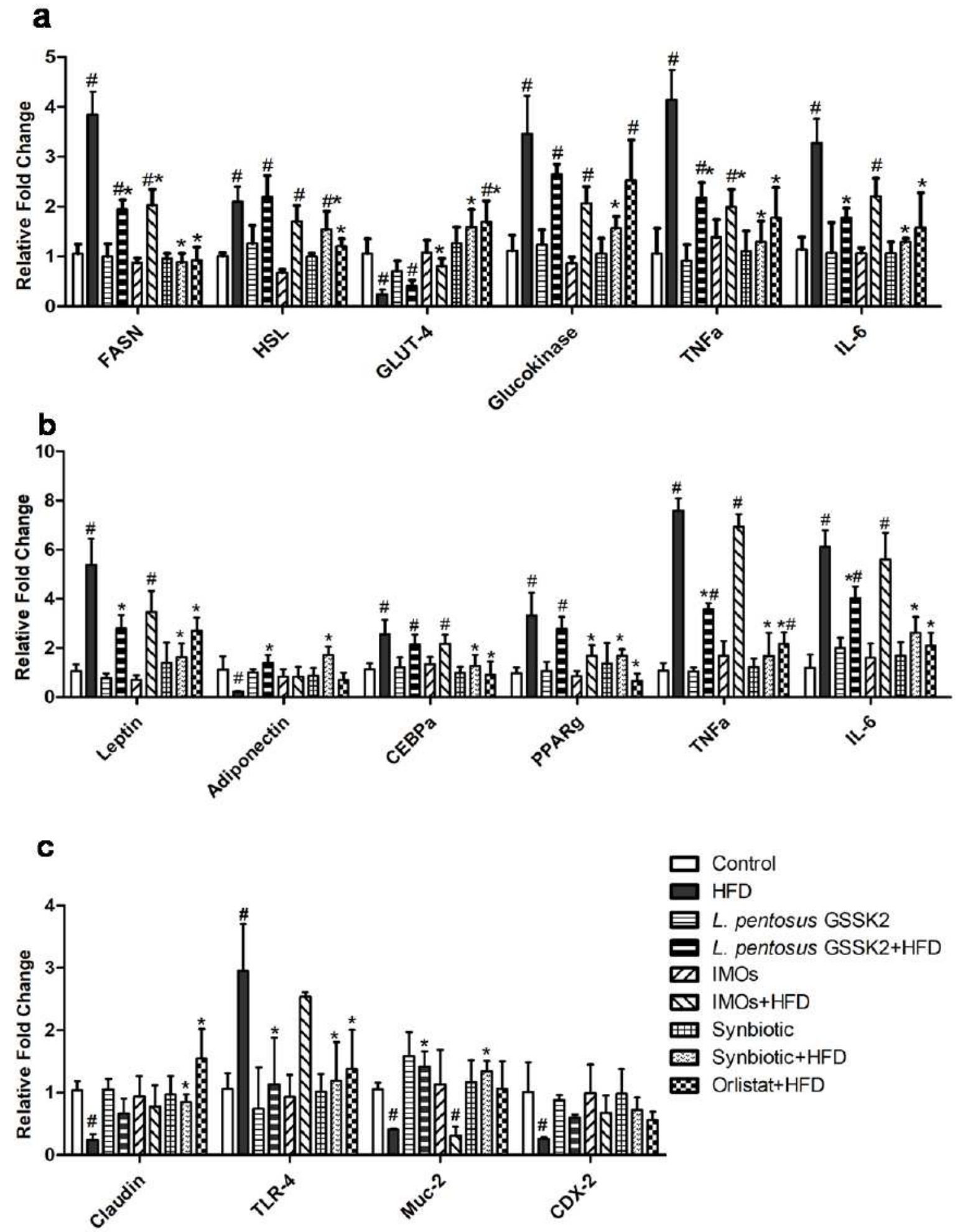

Figure 4

Relative gene expression of: (a) lipid metabolism genes, inflammatory markers, glucose metabolism genes in liver; (b) adiposity genes in adipose tissue; (c) gut integrity genes in colon of animals belonging to various groups by real-time PCR. Values are Mean $\pm S D, \# p<0.05$ versus control, ${ }^{*} p<0.05$ versus HFD. 

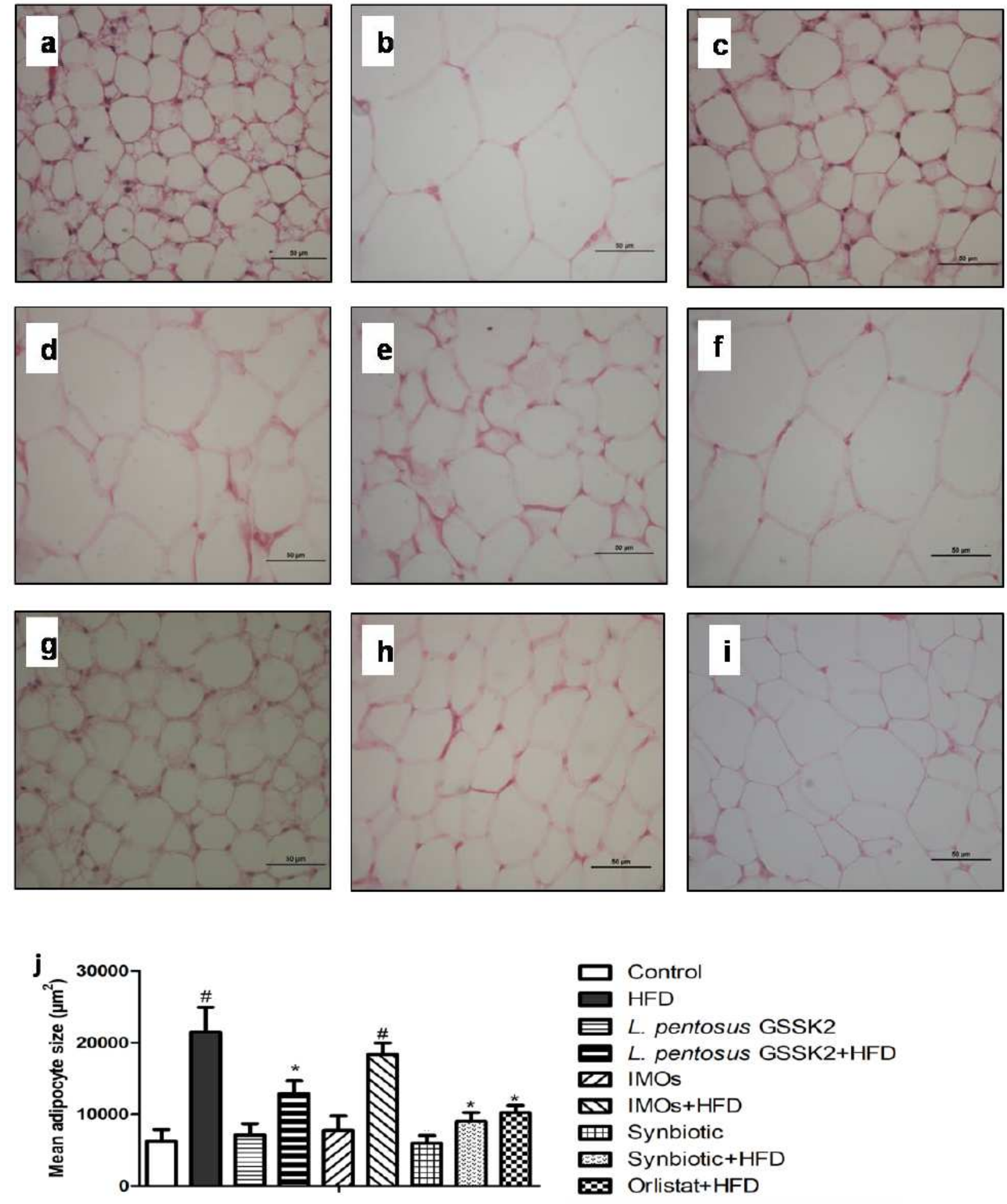

\section{Figure 5}

Photomicrograph of adipose tissue showing: (a) normal histomorphology with uniform, spherical adipocytes in control animals; (b) hypertrophied adipocytes in HFD; (c, e \& g) normal histoarchitecture of adipocytes in L. pentosus, IMOs and synbiotic animals; (d, f, h \& i) reduced adiposity in L. pentosus + HFD, IMOs + HFD, synbiotic + HFD and orlistat + HFD animals (H \& E staining; scale bar: $50 \mu \mathrm{m}, 400 \mathrm{X})$; (j) mean adipocyte size in animals belonging 695 to different groups. 

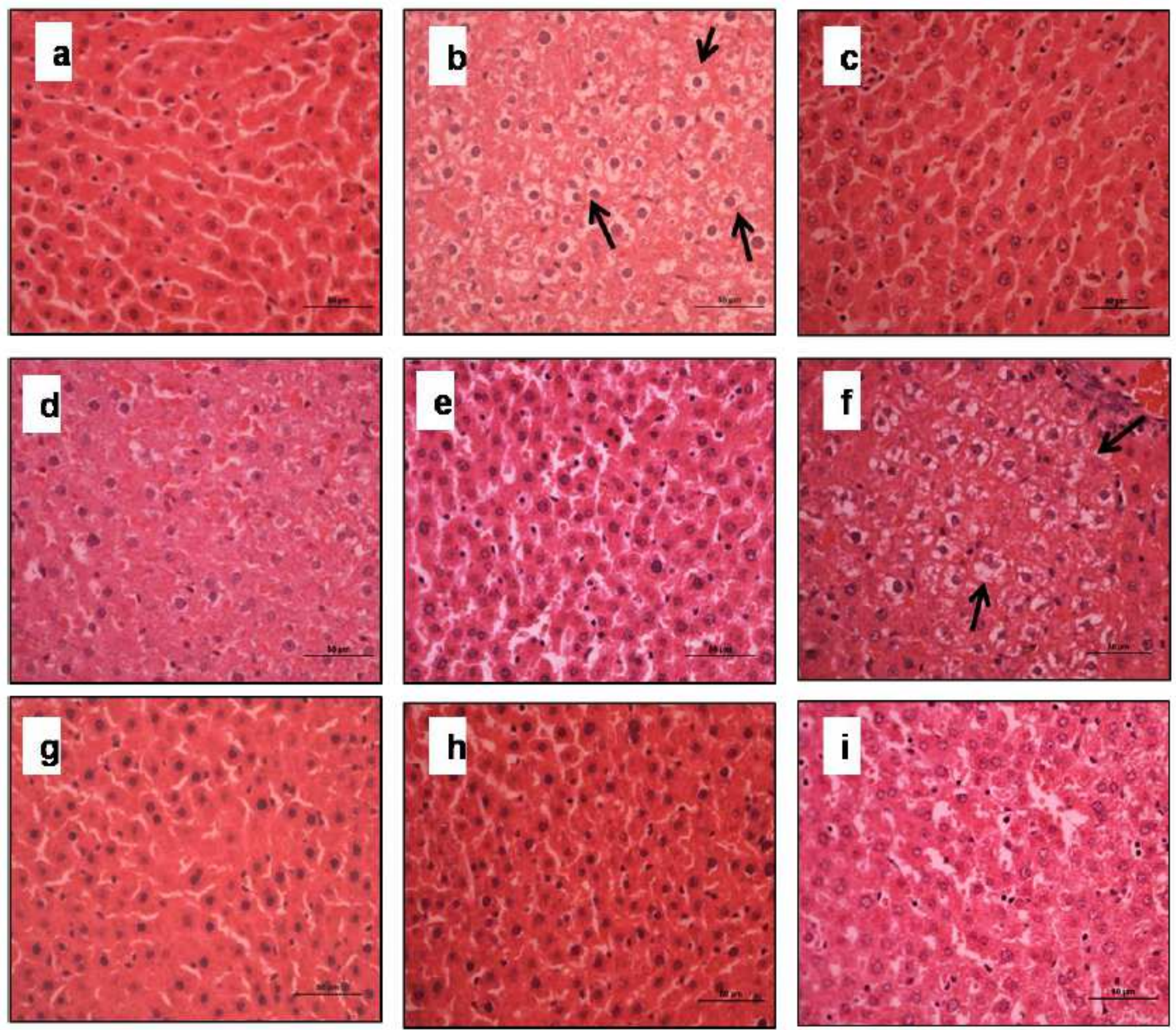

\section{Figure 6}

Photomicrograph of liver of animals belonging to different groups showing: (a) Normal histomorphology with polyhedral hepatocytes having large, rounded vesicular nuclei in control; (b) severe hepatic steatosis and ballooning degeneration of hepatocytes in HFD; (c, e \& g) normal histoarchitectureof hepatocytes in L. pentosus, IMOs and synbiotic animals; (f) ballooned hepatocytes with vacuolated nuclei in IMOs + HFD animals; (d, h \& i) reduced hepatic steatosis in L. pentosus + HFD, synbiotic + HFD and orlistat + HFD animals (H \& E staining; arrows indicate ballooned hepatocytes; scale bar: $50 \mu \mathrm{m}, 400 \mathrm{X}$ ). 

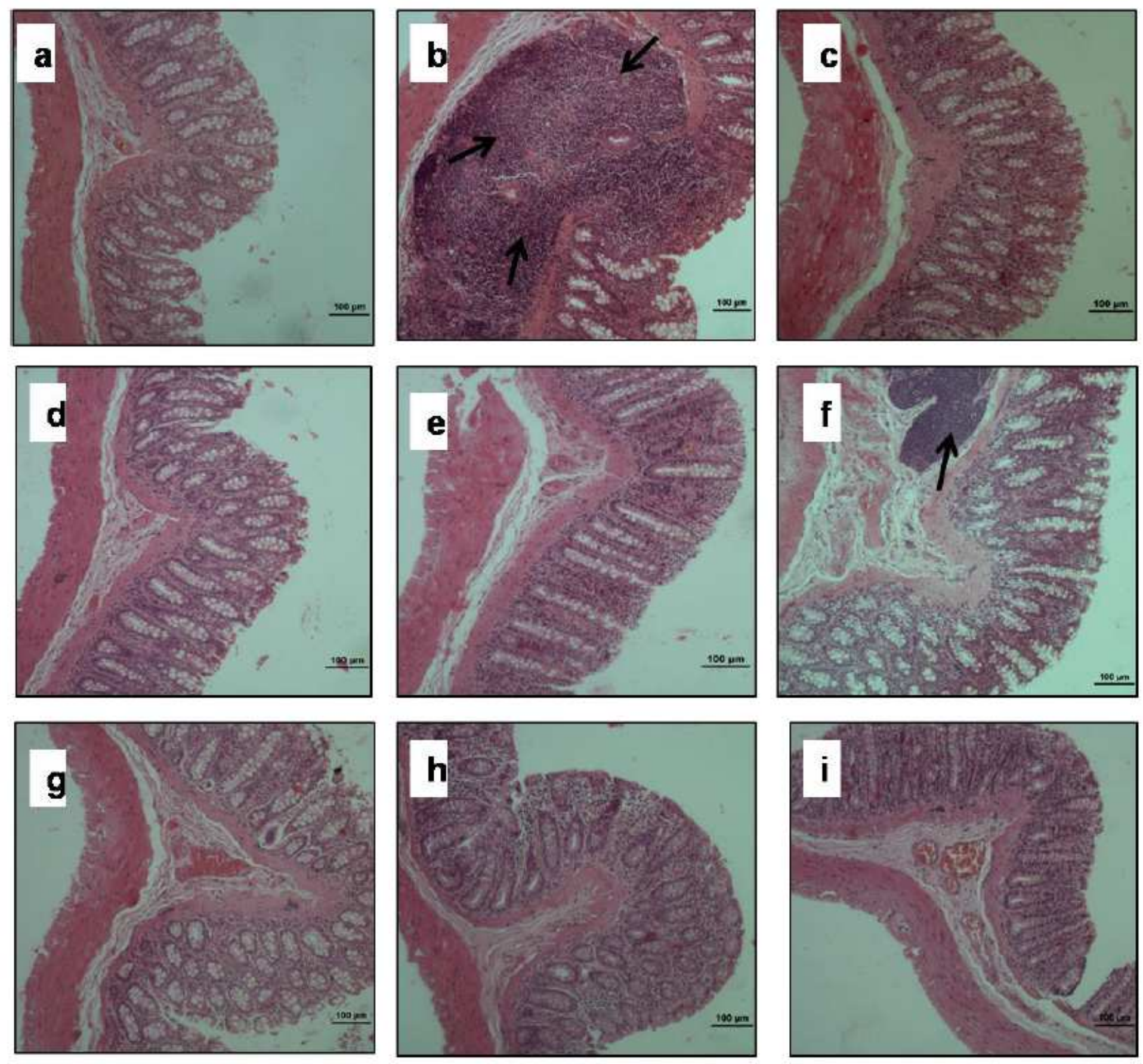

\section{Figure 7}

Photomicrograph of colon of animals belonging to different groups depicting: (a) normal histoarchitecture showing mucosa, submucosa, muscularis propria and serosa in control; (b) severely damaged mucosa with infilteration of lymphocytes and plasma cells in HFD; (c, e \& g) normal histomorphology of colon in L. pentosus, IMOs and synbiotic animals; (f) inflammation in IMOs + HFD animals; (d, h \& i) reduced infilteration of immune cells with intact mucosa in L. pentosus + HFD, synbiotic + HFD and orlistat + HFD animals (H \& E staining; arrows indicate infilteration of inflammatory cells; scale bar: $100 \mu \mathrm{m}, 100 \mathrm{X})$. 


\section{Supplementary Files}

This is a list of supplementary files associated with this preprint. Click to download.

- supplementarydata.pdf 